\title{
Analytical and Numerical Results for the Rational Large Eddy Simulation Model
}

\author{
David Barbato, Luigi C. Berselli* and Carlo R. Grisanti
}

Communicated by G. P. Galdi

\begin{abstract}
In this paper we analyze the Rational Large Eddy Simulation model. We start by introducing the system of partial differential equations we shall consider, together with its derivation. Then, we prove a result of full regularity for strong solutions in the space periodic setting. We also construct some exact solutions useful for the numerical benchmarking and finally we provide the results of some numerical experiments we performed.
\end{abstract}

Mathematics Subject Classification (2000). Primary 76D03; Secondary 35Q30, 76F65.

Keywords. Large Eddy Simulation, regularity, exact solutions, numerical simulations.

\section{Introduction}

In this paper we analyze the Rational Large Eddy (RLES in the sequel) model, introduced by Galdi and Layton [17]:

$$
\left\{\begin{aligned}
\frac{\partial w}{\partial t}+\nabla q+\nabla \cdot(w \otimes w)-\frac{1}{R e} \Delta w & \\
+\nabla \cdot\left(\mathrm{I}-\frac{\delta^{2}}{24} \Delta\right)^{-1}\left[\frac{\delta^{2}}{12} \nabla w \nabla w^{T}\right] & =\bar{f} \\
\nabla \cdot w & =0 \\
w(x, 0) & =w^{0}(x) .
\end{aligned}\right.
$$

Here, $R e>0$ is the Reynolds number, while the vector field $w: \Omega \times[0, T] \rightarrow \mathbf{R}^{3}$ is an approximation, formally of order $O\left(\delta^{4}\right)$, of $u$ that comes out by filtering the

${ }^{*}$ Corresponding author. 
Navier-Stokes equations

$$
\left\{\begin{aligned}
\frac{\partial u}{\partial t}+\nabla p+\nabla \cdot(u \otimes u)-\frac{1}{R e} \Delta u & =f, \\
\nabla \cdot u & =0, \\
u(x, 0) & =u^{0}(x),
\end{aligned}\right.
$$

that are the well-known equations describing the motion of viscous, incompressible fluids (as usual the Navier-Stokes equations are studied in $\Omega$ with homogeneous Dirichlet boundary conditions).

More precisely, $w$ is an approximation of

$$
\bar{u}(x, t)=g_{\delta}(x) * u(x, t),
$$

where $g_{\delta}(x)$ is a Gaussian kernel

$$
g_{\delta}(x)=\left(\frac{6}{\pi}\right)^{3 / 2} \frac{1}{\delta^{3}} \mathrm{e}^{-\frac{6|x|^{2}}{\delta^{2}}},
$$

while $*$ denotes the usual convolution.

The study of flow at high Reynolds number poses problems that, at present, make not possible a Direct Numerical Simulations (DNS) for realistic values of Re. Consequently it is necessary to find approximate equations, easier to handle from the numerical point of view.

The mathematical challenge is to find the equations satisfied by the filtered velocity $\bar{u}$ that should describe the "mean motion" of the fluid - roughly speaking the good part of the flow, without the fluctuation due to turbulent effects.

Our intent is to continue the mathematical analysis of the RLES model started in Iliescu [21], Berselli et al. [8], and Berselli and Grisanti [9], and also to provide some numerical tests for the pure Fourier-Galerkin method.

We recall that the model (1.1) is derived with the classical methods of Large Eddy Simulation (LES). We also point out that the system appearing in (1.1) is not a differential system, due to the presence of the non-local term

$$
\left(\mathrm{I}-\frac{\delta^{2}}{24} \Delta\right)^{-1}\left[\frac{\delta^{2}}{12} \nabla w \nabla w^{T}\right]_{i j}:=\left(\mathrm{I}-\frac{\delta^{2}}{24} \Delta\right)^{-1} \frac{\delta^{2}}{12} \sum_{l=1}^{3} \frac{\partial w^{i}}{\partial x_{l}} \frac{\partial w^{j}}{\partial x_{l}} .
$$

In this context the appropriate boundary conditions that should be supplemented in the inversion of the Laplace operator are not completely known, and the various tests performed herein and in references [22, 23, 25, 27] regard the periodic case or the Neumann boundary conditions.

The problem of the boundary condition that should complete system (1.1) is still open. We consider the periodic setting, that is the simplest to threat and the only one that allow us to prove rigorous results. We observe that a Navier-type condition has been proposed by Galdi and Layton [17] and a recent study also of nonlinear slip with friction boundary conditions can be found in John, Layton, and Sahin [28]. 
For the sake of completeness, we want to point out that the RLES is one of the possible LES models that has been proposed. In particular, we want to compare our results with others obtained for the same model. We apologize, but references are necessarily related only to a restricted part of the relevant work on the subject. For recent developments - along different paths - we refer the interested reader to the following papers (in alphabetic order): Aldama [3]; Cottet, Jiroveanu, and Micheaux [14]; Foiaş, Holm, and Titi [16]; Hughes, Mazzei and Jansen [20]; Stolz and Adams [36], and many many others, cited for instance in the recent book on the subject written by Sagaut [35] and Geurts [18]. Several papers can be found on LES and we are just citing those regarding models similar to ours, and those using the techniques typical of the mathematical and numerical analysis of partial differential equations.

Plan of the paper. In Section 2 we briefly derive the RLES model and we give some references to other method recently proposed or commonly used. In Section 3 we recall some of the known analytical results, and we prove a regularity result. In Section 4 we provide the study of some classes of exact solutions and in Section 5 we report the results of some numerical experiments.

\section{Derivation of RLES the model}

For the reader's convenience, we recall the main steps and assumption needed to derive system (1.1), approximating the mean velocity.

The procedure can be resumed in the following steps and, apart step number 5, the technique is typical of other classic LES models.

1. extend all the variables appearing in the Navier-Stokes equations (1.2) to $\overrightarrow{0}$ outside the domain $\Omega$;

2. apply the filter, that acts as a convolution with the gaussian kernel $g_{\delta}$;

3. assume that convolution and the linear operators commute;

4. pass to the frequency space via the Fourier transform;

5. by using the so called $(\mathbf{0 , 1})$ subdiagonal Padé rational approximation of the exponential function, approximate the Fourier transform of $\overline{u \otimes u}=$ $g_{\delta} *\left(u_{i} u_{j}\right)$ in terms only of the Fourier transform of $\bar{u}=g_{\delta} * u$. This is done up to a certain order of $\delta$;

6. apply the inverse Fourier Transform to get system (1.1).

This way of reasoning leads to the system (1.1) that does not contain $O\left(\delta^{4}\right)$ terms. Terms that involving higher powers of $\delta$ are supposed to be small, compared to those that are retained. This is just a formal procedure, since nothing ensures that the disregarded terms are smaller than the retained ones. On the other hand, this is the starting step in the derivation of reasonable LES models and we do not insist on it. For further details and comprehensive references on classical LES models based on wave-number asymptotic, see Aldama [3] and the recent books by Sagaut [35] and John [27]. 
The subdiagonal approximation is used to approximate the Fourier transform of the Gaussian kernel in a satisfactory physical consistent way. The complete derivation of the RLES model (1.1), together with the physical motivation that inspired it can be found in Galdi and Layton [17].

Remark 2.1. We observe that the derivation is based on the application of the Fourier Transform and then in presence of boundaries, the functions are simply extended by zero outside their domain. This introduce an additional Boundary Commutation Error, as reported and analyzed by Dunca, John, and Layton [15].

To be more precise in the description of the derivation of the model, we briefly explain the steps that lead to (1.1) and also to the gradient model.

\subsection{The gradient model}

Since we assume that $u$ is equal to its filtered part $\bar{u}$, plus "turbulent fluctuations" (defined by $u^{\prime}=u-\bar{u}$ ) we have

$$
u=\bar{u}+u^{\prime} \quad \text { and } \quad \bar{u}=g *\left(\bar{u}+u^{\prime}\right)
$$

and an application of the Fourier transform yields $\widehat{\bar{u}}(k)=\widehat{g}_{\delta}(k) \widehat{\bar{u}}(k)+\widehat{g}_{\delta}(k) \widehat{u^{\prime}}(k)$, with

$$
\widehat{u^{\prime}}(k)=\left(\frac{1}{\widehat{g}_{\delta}(k)}-1\right) \widehat{\bar{u}}(k)
$$

The above formula gives

$$
\begin{aligned}
& \widehat{\overline{\bar{u}}}=\widehat{g}_{\delta}(k) \widehat{\bar{u}}(k) * \widehat{\bar{u}}(k) \\
& \widehat{\overline{\bar{u} u^{\prime}}}=\widehat{g}_{\delta}(k) \widehat{\bar{u}}(k) *\left[\left(\frac{1}{\widehat{g}_{\delta}(k)}-1\right) \widehat{\bar{u}}(k)\right] \\
& \widehat{\widehat{u^{\prime} \bar{u}}}=\widehat{g}_{\delta}(k)\left[\left(\frac{1}{\widehat{g}_{\delta}(k)}-1\right) \widehat{\bar{u}}(k)\right] * \widehat{\bar{u}}(k) \\
& \widehat{\overline{u^{\prime} u^{\prime}}}=\widehat{g}_{\delta}(k)\left[\left(\frac{1}{\widehat{g}_{\delta}(k)}-1\right) \widehat{\bar{u}}(k)\right] *\left[\left(\frac{1}{\widehat{g}_{\delta}(k)}-1\right) \widehat{\bar{u}}(k)\right]
\end{aligned}
$$

Some conventional LES models are derived by using the following Taylor series expansion for $\widehat{g}_{\delta}(k)$ :

$$
\widehat{g}_{\delta}(k)=1-\frac{\delta^{2}}{4 \gamma}|k|^{2}+O\left(\delta^{4}\right), \quad \frac{1}{\widehat{g}_{\delta}(k)}-1=\frac{\delta^{2}}{4 \gamma}|k|^{2}+O\left(\delta^{4}\right)
$$


Substitution of (2.1) in the above formulas, and application of the inverse Fourier transform implies the following expressions:

$$
\begin{aligned}
\overline{\bar{u}} \bar{u} & =\bar{u} \bar{u}+\frac{\delta^{2}}{4 \gamma} \Delta(\bar{u} \bar{u})+O\left(\delta^{4}\right) \\
\overline{\bar{u} u^{\prime}} & =-\frac{\delta^{2}}{4 \gamma} \bar{u} \Delta \bar{u}+O\left(\delta^{4}\right) \\
\overline{u^{\prime} \bar{u}} & =-\frac{\delta^{2}}{4 \gamma} \Delta \bar{u} \bar{u}+O\left(\delta^{4}\right) \\
\overline{u^{\prime} u^{\prime}} & =O\left(\delta^{4}\right) .
\end{aligned}
$$

Ignoring the terms that are of order of $\delta^{4}$, we finally get the "gradient model"

$$
\left\{\begin{aligned}
\frac{\partial w}{\partial t}+\nabla q+\nabla \cdot(w \otimes w)-\frac{1}{R e} \Delta w+\nabla \cdot\left[\frac{\delta^{2}}{12} \nabla w \nabla w^{T}\right] & =\bar{f}, \\
\nabla \cdot w & =0 \\
w(x, 0) & =w^{0}(x) .
\end{aligned}\right.
$$

Note that this classical method has the drawback of a possible increasing of high wave-numbers. In fact, if $u \in L^{2}$ we cannot ensure that $\widehat{w}=\widehat{g_{\delta} * u} \simeq\left(1-\frac{\delta^{2}}{4 \gamma}\right)|k|^{2} \widehat{u}$ belongs again to $L^{2}$. Anyway it has been employed quite successfully in conjunction with additional dissipation for subfilter scale terms, see [13, 25, 14]

\subsection{The Rational LES model}

The RLES model (1.1) is derived along the same lines, but by using the following approximation. The Padé approximant are introduced since they are decreasing at infinity and the corresponding approximate equations (approximate to the order of $\delta^{4}$ ) may have a better behavior.

By using the following $(0,1)$-Padé approximation of the exponential

$$
\widehat{g}_{\delta}(k)=\frac{1}{1+\frac{\delta}{4 \gamma}|k|^{2}}+O\left(\delta^{4}\right), \quad \frac{1}{\widehat{g}_{\delta}(k)}-1=\frac{\delta}{4 \gamma}|k|^{2}+O\left(\delta^{4}\right)
$$

and by using the same formal techniques used in the derivation of the Gradient model (2.2), we obtain corresponding terms:

$$
\begin{aligned}
& \overline{\bar{u}} \bar{u}=\left(\mathrm{I}-\frac{\delta^{2}}{24} \Delta\right)^{-1} \bar{u} \bar{u}+O\left(\delta^{4}\right) \\
& \overline{\bar{u} u^{\prime}}=-\frac{\delta^{2}}{4 \gamma}\left(\mathrm{I}-\frac{\delta^{2}}{24} \Delta\right)^{-1} \bar{u} \Delta \bar{u}+O\left(\delta^{4}\right) \\
& \overline{u^{\prime} \bar{u}}=-\frac{\delta^{2}}{4 \gamma}\left(\mathrm{I}-\frac{\delta^{2}}{24} \Delta\right)^{-1} \Delta \bar{u} \bar{u}+O\left(\delta^{4}\right)
\end{aligned}
$$




$$
\overline{u^{\prime} u^{\prime}}=\frac{\delta^{4}}{16 \gamma^{2}}\left(\mathrm{I}-\frac{\delta^{2}}{24} \Delta\right)^{-1} \Delta \bar{u} \Delta \bar{u}+O\left(\delta^{6}\right)
$$

Substitution into the equation and some algebraic manipulations give finally system (1.1).

In the above system we did not specify the boundary conditions involved in the inversion of the Laplace operator. In fact, there are no conditions working in the whole space. In the applications it is standard to employ the Neumann ones (the natural for the Laplace equations), or the periodic ones, in the space periodic setting.

\subsection{Various remarks}

We observe that the RLES model is very similar to the Lagrangian Averaged Navier-Stokes (LANS) $\alpha$-model (that has been derived in a completely different way) introduced by Holm et al. [19], and recently analyzed by Foiaş et al. [16].

From the point of view of modeling we note that both the Gradient and the RLES models discard the subfilter scale terms

$$
(u-\bar{u}) \otimes(u-\bar{u}) \stackrel{\text { def }}{=} u^{\prime} \otimes u^{\prime},
$$

since they turn out to be (formally) of the fourth order, in that development. For this reason, other high order Rational LES models (HOSFS), that involve high order Padé approximants, have been recently investigated, see Berselli and Iliescu [10]. Other high order LES models, derived in a different way, have been recently proposed; see for instance Katopodes et al. [29].

Passing to some numerical results, we recall that the solutions to (1.1) show a better behavior, with respect to other models commonly used in LES. In this respect see the comparison of various model performed by Iliescu et al. [25].

The problem of the "closure" of the system has been studied by several authors. By closure we mean to find the differential (or integro-differential or pseudodifferential, or something else) system satisfied by $\bar{u}$, i.e., a system having only $(\bar{u}, \bar{p})$ as unknowns. A classical system that should model the effect of the unresolved scales, i.e., of the terms that are not retained, is the model proposed by Smagorinsky (with $\mu=1$ ):

$$
\left\{\begin{aligned}
\frac{\partial w}{\partial t}+\nabla p+\nabla \cdot(w \otimes w)-\frac{1}{R e} \Delta w- & \\
-c_{S} \nabla \cdot\left(\left|\nabla w+\nabla w^{T}\right|^{2 \mu}\left(\nabla w+\nabla w^{T}\right)\right) & =f, \\
\nabla \cdot w & =0, \\
w(x, 0) & =w^{0}(x) .
\end{aligned}\right.
$$

This system has been introduced in the meteorology context and it is based on the physical assumption (assumption made by Boussinesq in 1877 for the first 
time) that turbulent fluctuations are "dissipative in mean." System (2.4) results too dissipative for applications, but a combination of the gradient model and an extra-dissipative term is widely used in the application: it is a common practice to use $p$-Laplacian to stabilize the numerical simulations, obtaining a class of mixed models. The Smagorinsky system had some interest also in the study of the possible regularity of the Navier-Stokes equations, considering the limit with $\mu \rightarrow 0$. The mathematical analysis for system (2.4) started with Ladyžhenskaya [31].

\section{Analytical results}

A preliminary analysis, concerning the possible existence of weak solution for (1.1), has been performed by Galdi, Iliescu, and Layton (see Iliescu [21]). By adding an extra dissipative term of the Smagorinsky type

$$
\left(c_{1}+c_{2}\left|\nabla \bar{u}+\nabla \bar{u}^{\mathrm{T}}\right|^{2 \mu}\right)\left(\nabla \bar{u}+\nabla \bar{u}^{\mathrm{T}}\right) .
$$

with $c_{1}, c_{2}$ positive constants and $\mu \geq 0.1$, they obtained suitable energy estimates. See also the discussion in John [27]. The results are similar to those obtained by Coletti [12] for the gradient model.

Here and in the sequel we consider periodic functions and consequently $Q=$ ] $0, \mathcal{L}\left[{ }^{3} \subset \mathbf{R}^{3}\right.$; besides $H^{s}$ will denote the Hilbert space of periodic vector valued functions $u$, belonging to the Sobolev space $\left[H^{s}(Q)\right]^{3}$ and with vanishing mean value. The functions in $H^{s}$ can be written as

$$
u=\sum_{k \in \mathbf{Z}^{3}} c_{k} \mathrm{e}^{\frac{2 i \pi k \cdot x}{\mathcal{L}}}, \quad \bar{c}_{k}=c_{-k}, \quad c_{0}=0
$$

and the norm in $H^{s}$ is defined as

$$
\|u\|_{H^{s}}^{2}=\sum_{k \in \mathbf{Z}^{3}}|k|^{2 s}\left|c_{k}\right|^{2} .
$$

Note that the latter formula allows to consider real (and also negative) values for $s$. The spaces $L^{p}, 1 \leq p \leq \infty$, with norm $\|\cdot\|_{p}$ are the customary Lebesgue spaces on $Q$; we also denote by $W^{k, p}$, with norm $\|\cdot\|_{W^{k, p}}$, the usual Sobolev spaces on $Q$ and we do not distinguish between scalar and vector valued functions spaces. We use this particular simple setting to avoid the big technical difficulties due to the boundary conditions. In this way we can focus on fine properties of the solutions to $(1.1)$.

In reference [8] it is analyzed system (1.1) (i.e., no additional Smagorinsky terms are added) and it is proved the following result of existence and uniqueness of strong solutions, i.e., solutions such that

$$
w \in L^{\infty}\left(0, T ; H^{1}\right) \cap L^{2}\left(0, T ; H^{2}\right) \quad \text { and } \quad \frac{\partial w}{\partial t} \in L^{2}\left(0, T ; L^{2}\right) .
$$


Theorem 3.1. Let be given $w_{0} \in H^{1}$, with $\nabla \cdot w_{0}=0$, and let be given $\bar{f} \in$ $L^{2}\left(0, T ; L^{2}\right)$. Then, there exists a strictly positive $T^{*}=T^{*}\left(\left\|\nabla w_{0}\right\|_{2}, R e, \bar{f}, \delta\right)$ such that there exists a unique strong solution to (1.1) in $\left[0, T^{*}\right)$.

The proof is based on a Galerkin approximation and standard tools of functional analysis, together with precise estimates of the turbulent part of the stress tensor - the one coming out from the term (1.3) - in negative Sobolev spaces.

This result has been improved in Berselli and Grisanti [9], where it is shown that $T^{*}$ is, in fact, independent of $\delta$ :

Theorem 3.2. The life span of a strong solution to the RLES model depends on $\left\|\nabla w_{0}\right\|_{L^{2}}, R e$, and $\|\bar{f}\|_{L^{2}\left(0, T ; L^{2}\right)}$, but it is independent of $\delta$.

The above theorem derives from sharp estimates on the Laplace operator and on the nonlinear terms appearing in (1.3).

By using this fact in [9] it is also proved that the RLES model is consistent, i.e., that strong solutions to the RLES model converge to strong solutions to the Navier-Stokes equations, as $\delta \rightarrow 0$.

Theorem 3.3. Let $w$ be a strong solution (we do not write it explicitly, but it depends on $\delta$ ) to (1.1), while let $u$ be a strong solution to the Navier-Stokes equations, in the common time interval $[0, T] . w(x, 0)$ and $u(x, 0)$ belong also to $H^{2}$ and

$$
\exists c_{1}>0: \quad\|w(x, 0)-u(x, 0)\|_{L^{2}} \leq c_{1} \delta^{2} .
$$

Then we have, for some $c_{2}>0$,

$$
\sup _{t \in[0, T]}\|w(x, t)-u(x, t)\|_{L^{2}} \leq c_{2} \delta^{2} .
$$

If, in addition,

$$
\exists c_{3}>0: \quad\|w(x, 0)-u(x, 0)\|_{H^{1}} \leq c_{3} \delta,
$$

then we have, for some $c_{4}>0$,

$$
\sup _{t \in[0, T]}\|w(x, t)-u(x, t)\|_{H^{1}} \leq c_{4} \delta .
$$

Remark 3.4. To our knowledge, only for the "LES scale similarity model" studied in Layton [33] consistency was known. The issue of consistency (together with that of "sensitivity") is nowadays becoming central in LES and consistency of some methods have been recently proved by Kaya and Layton, Holm, Foiaş, and Titi, Layton and Lewandowski.

The proof of Theorem 3.3 is essentially based on a regularity result. In particular, it is based on the first inductive step of the following result, that is the main functional result of this paper 
Theorem 3.5. Let $w$ be a strong solution to (1.1) in $\left[0, T^{*}\left[\right.\right.$. If $\bar{f} \in C^{\infty}$, then

$$
w \in C^{\infty}(] 0, T^{*}[\times Q) .
$$

Proof. Note that in the sequel we keep $\delta>0$ fixed, but the various constant that appear in the sequel may depend on $\delta$.

For simplicity we set $\bar{f}=0$. The general case follows with minor changes. We will use the following notations: we set $c_{\delta}:=\delta^{2} / 24$ and, given a square matrix $A$, we write $\mathbf{D}(A):=\frac{A+A^{T}}{2}$.

First, we prove some lemmata.

Lemma 3.6. Let $\Omega \subset \mathbf{R}^{n}$ be a bounded domain with smooth boundary. Then, for every vector field $F \in W^{k, p}(\Omega), 1<p<\infty, k>-1$ there exist one and only one couple of functions $(u, \phi)$ solving

$$
-\frac{1}{R e} \Delta u=\nabla \phi+F
$$

and $\int_{\Omega} \phi d x=0, \nabla \cdot u=0, u \in W^{k+2, p}(\Omega) \cap W_{0}^{1, p}(\Omega), \phi \in W^{k+1, p}(\Omega)$. Moreover, the following estimate holds:

$$
\|u\|_{W^{k+2, p}}+\|\phi\|_{W^{k+1, p}} \leq c\|F\|_{W^{k, p}}
$$

where $c$ is a positive constant independent of $(u, \phi)$.

Lemma 3.7. Let $w$ be a strong solution to (1.1) in $\left[0, T^{*}[\right.$. Then, for every $k \in \mathbf{N}$ and for every $\eta>0, D_{t}^{k} w \in C\left(\left[\eta, T^{*}\left[; H^{2}(Q)\right)\right.\right.$.

Proof. We shall prove the thesis by induction on $k \in \mathbf{N}$. More precisely we want to prove the following inductive statement:

1. $D_{t}^{k} w \in L^{\infty}\left(\eta, T^{*} ; H^{1}(Q)\right) \cap L^{2}\left(\eta, T^{*} ; H^{2}(Q)\right)$;

2. $D_{t}^{k+1} w \in L^{2}\left(\eta, T^{*} ; L^{2}(Q)\right)$.

The initial step for $k=0$ has been proved in the existence Theorem 3.1. We want to show the step with $k=1$. Here and in the following estimates we are working with the Galerkin approximations for $w$, which, for simplicity, will be written as $w$ instead of $w_{n}$. The solution $w$ will inherit the regularity from the uniform estimates we can prove for the Galerkin approximation $w_{n}$.

Let us derive the equations of the RLES model with respect to $t$ :

$$
\begin{aligned}
w_{t t}-\frac{1}{R e} \Delta w_{t}= & -\left(w_{t} \cdot \nabla\right) w-(w \cdot \nabla) w_{t}+\nabla q_{t}- \\
& -\nabla \cdot\left(\mathrm{I}-\frac{\delta^{2}}{24} \Delta\right)^{-1} \frac{\delta^{2}}{12}\left[\nabla w_{t} \nabla w^{T}+\nabla w \nabla w_{t}^{T}\right] .
\end{aligned}
$$


Multiplying the above equation by $w_{t}$ and integrating over $Q$ we obtain:

$$
\begin{aligned}
\frac{1}{2} & \frac{d}{d t}\left\|w_{t}\right\|_{2}^{2}+\frac{1}{R e}\left\|\nabla w_{t}\right\|_{2}^{2} \\
= & -\int_{Q}\left(w_{t} \cdot \nabla\right) w \cdot w_{t} d x-\int_{Q}(w \cdot \nabla) w_{t} \cdot w_{t} d x \\
& -\int_{Q} \nabla \cdot\left(\mathrm{I}-c_{\delta} \Delta\right)^{-1} 4 c_{\delta} \mathbf{D}\left(\nabla w_{t} \nabla w^{T}\right) \cdot w_{t} d x \\
= & -\int_{Q}\left(w_{t} \cdot \nabla\right) w \cdot w_{t} d x+\int_{Q}\left(\mathrm{I}-c_{\delta} \Delta\right)^{-1} 4 c_{\delta} \mathbf{D}\left(\nabla w_{t} \nabla w^{T}\right) \cdot \nabla w_{t} d x .
\end{aligned}
$$

Let us estimate the last two integrals. By using Hölder inequality and the Sobolev embeddings $W^{2,2} \subset W^{1,3}, W^{1,2} \subset L^{6}$ (we are working in a subset of $\mathbf{R}^{3}$ ) we have:

$$
\left|\int_{Q}\left(w_{t} \cdot \nabla\right) w \cdot w_{t} d x\right| \leq\left\|w_{t}\right\|_{2}\|\nabla w\|_{3}\left\|w_{t}\right\|_{6} \leq c\left\|w_{t}\right\|_{2}\|w\|_{W^{2,2}}\left\|\nabla w_{t}\right\|_{2} .
$$

Now we use Helmholtz decomposition to write $\Delta w=P \Delta w+\nabla \phi$ (where $P$ is the orthogonal projector over divergence free functions) and we use Lemma 3.6, obtaining $\|w\|_{W^{2,2}} \leq c\|P \Delta w\|_{2}$. So, for every $\epsilon>0$, by the Schwartz inequality, we have:

$$
\left\|w_{t}\right\|_{2}\|w\|_{W^{2,2}}\left\|\nabla w_{t}\right\|_{2} \leq \frac{c}{2 \epsilon}\left\|w_{t}\right\|_{2}^{2}\|P \Delta w\|_{2}^{2}+\frac{c \epsilon}{2}\left\|\nabla w_{t}\right\|_{2}^{2}
$$

Now we need an estimate for $\|P \Delta w\|_{2}$. Multiplying equation (1.1) by $P \Delta w$ and integrating over $Q$ we obtain:

$$
\begin{aligned}
\int_{Q} w_{t} P \Delta w d x- & \frac{1}{R e}\|P \Delta w\|_{2}^{2}+\int_{Q}(w \cdot \nabla) w P \Delta w d x \\
& +\int_{Q} \nabla \cdot\left(\mathrm{I}-c_{\delta} \Delta\right)^{-1} 2 c_{\delta}\left(\nabla w \nabla w^{T}\right) \cdot P \Delta w d x=0 .
\end{aligned}
$$

We use Hölder inequality and the interpolation $L^{3}=\left[L^{2} ; L^{6}\right]_{1 / 2}$ to evaluate the following term:

$$
\left|\int_{Q}(w \cdot \nabla) w P \Delta w d x\right| \leq\|w\|_{6}\|\nabla w\|_{3}\|P \Delta w\|_{2} \leq\|w\|_{6}\|\nabla w\|_{2}^{\frac{1}{2}}\|\nabla w\|_{6}^{\frac{1}{2}}\|P \Delta w\|_{2} .
$$

By the Sobolev embedding $W^{2,2} \hookrightarrow W^{1,6}$, Lemma 3.6, and the inductive hypothesis - which ensures $w \in L^{\infty}\left(\epsilon, T^{*} ; L^{6}(Q)\right)$ - we obtain the following bound:

$$
\left|\int_{Q}(w \cdot \nabla) w \cdot P \Delta w d x\right| \leq c\|\nabla w\|_{2}^{1 / 2}\|P \Delta w\|_{2}^{3 / 2} \leq K(\epsilon)\|\nabla w\|_{2}^{2}+\epsilon\|P \Delta w\|_{2}^{2},
$$

with $K(\epsilon)$ suitable constant independent on $t$. Moreover, using the Sobolev embedding $W^{2,6 / 5} \hookrightarrow H^{1}$ and the continuity of the gradient operator between $L^{2}$ 
and $H^{-1}$, we have:

$$
\begin{aligned}
& \left|\int_{Q} \nabla \cdot\left(\mathrm{I}-c_{\delta} \Delta\right)^{-1} 2 c_{\delta}\left(\nabla w \nabla w^{T}\right) \cdot P \Delta w d x\right| \\
& =2 c_{\delta}\left|\int_{Q}\left(\mathrm{I}-c_{\delta} \Delta\right)^{-1}\left(\nabla w \nabla w^{T}\right) \nabla P \Delta w d x\right| \\
& \leq 2 c_{\delta}\left\|\left(\mathrm{I}-c_{\delta} \Delta\right)^{-1}\left(\nabla w \nabla w^{T}\right)\right\|_{H^{1}}\|\nabla P \Delta w\|_{H^{-1}} \\
& \leq c_{\delta}^{\prime}\left\|\left(\mathrm{I}-c_{\delta} \Delta\right)^{-1}\left(\nabla w \nabla w^{T}\right)\right\|_{W^{2,6 / 5}}\|P \Delta w\|_{2} \leq c_{\delta}^{\prime \prime}\left\|\left(\nabla w \nabla w^{T}\right)\right\|_{6 / 5}\|P \Delta w\|_{2} .
\end{aligned}
$$

The last inequality is obtained by using Agmon-Douglis-Nirenberg regularity results, see $[1,2]$. In the sequel we will use the generic notation $c$ to indicate different constant, keeping in mind that they may depend on $\delta$, but this is not relevant in the present context. Now, we use Hölder inequality, the interpolation $L^{\frac{12}{5}}=\left[L^{2} ; L^{6}\right]_{3 / 4}$, the Sobolev embedding $W^{1,2} \hookrightarrow L^{6}$, and Lemma 3.6 to obtain:

$$
\begin{aligned}
\left\|\left(\nabla w \nabla w^{T}\right)\right\|_{6 / 5} & \leq c\|\nabla w\|_{12 / 5}^{2} \leq c\|\nabla w\|_{2}^{3 / 2}\|\nabla w\|_{6}^{1 / 2} \\
& \leq c\|\nabla w\|_{2}^{3 / 2}\left\|D^{2} u\right\|_{2}^{1 / 2} \leq c\|\nabla w\|_{2}^{3 / 2}\|P \Delta u\|_{2}^{1 / 2} .
\end{aligned}
$$

Substituting this result in (3.6) we have:

$$
\left|\int_{Q} \nabla \cdot\left(\mathrm{I}-c_{\delta} \Delta\right)^{-1} 2 c_{\delta}\left(\nabla w \nabla w^{T}\right) \cdot P \Delta w d x\right| \leq c\|\nabla w\|_{2}^{3 / 2}\|P \Delta w\|_{2}^{3 / 2} .
$$

Gathering together equation (3.5) and inequalities (3.6)-(3.7), we obtain the required estimate for $P \Delta w$ :

$$
\begin{aligned}
\|P \Delta w\|_{2}^{2} & \leq \operatorname{Re}\left(\left\|w_{t}\right\|_{2}\|P \Delta w\|_{2}+K(\epsilon)\|\nabla w\|_{2}^{2}+\epsilon\|P \Delta w\|_{2}^{2}+c\|\nabla w\|_{2}^{\frac{3}{2}}\|P \Delta w\|_{2}^{\frac{3}{2}}\right) \\
& \leq c\left(\frac{\left\|w_{t}\right\|_{2}^{2}}{\epsilon}+2 \epsilon\|P \Delta w\|_{2}^{2}+K(\epsilon)\|\nabla w\|_{2}^{2}+\frac{M(\epsilon)}{4}\|\nabla w\|_{2}^{6}+\frac{3}{4} \epsilon\|P \Delta w\|_{2}^{2}\right) \\
& =c_{1} \epsilon\|P \Delta w\|_{2}^{2}+c_{2}(\epsilon)\left(\left\|w_{t}\right\|_{2}^{2}+\|\nabla w\|_{2}^{2}+\|\nabla w\|_{2}^{6}\right) .
\end{aligned}
$$

Then, choosing $\epsilon$ small enough, it results:

$$
\|P \Delta w\|_{2}^{2} \leq c\left(1+\left\|w_{t}\right\|_{2}^{2}+\|\nabla w\|_{2}^{6}\right) \leq c_{1}\left(1+\left\|w_{t}\right\|_{2}^{2}\right)
$$

by the previous part of the inductive hypothesis, which ensures that $\|\nabla w\|_{2}$ is bounded uniformly with respect to $t$. By using inequalities (3.3), (3.4), and (3.8) we have:

$$
\begin{aligned}
\left|\int_{Q}\left(w_{t} \cdot \nabla\right) w \cdot w_{t} d x\right| & \leq \frac{c}{\epsilon}\left\|w_{t}\right\|_{2}^{2}\|P \Delta w\|_{2}^{2}+\epsilon\left\|\nabla w_{t}\right\|_{2}^{2} \\
& \leq \frac{c_{1}}{\epsilon}\left\|w_{t}\right\|_{2}^{2}\left(1+\left\|w_{t}\right\|_{2}^{2}\right)+\epsilon\left\|\nabla w_{t}\right\|_{2}^{2} \\
& \leq \frac{c_{2}}{\epsilon}\left(1+\left\|w_{t}\right\|_{2}^{4}\right)+\epsilon\left\|\nabla w_{t}\right\|_{2}^{2} .
\end{aligned}
$$


Now we evaluate the last integral in equation (3.2):

$$
\begin{aligned}
& \left|\int_{Q}\left(\mathrm{I}-c_{\delta} \Delta\right)^{-1} 4 c_{\delta} \mathbf{D}\left(\nabla w_{t} \nabla w^{T}\right) \cdot \nabla w_{t} d x\right| \\
& \leq\left\|\left(\mathrm{I}-c_{\delta} \Delta\right)^{-1} 4 c_{\delta} \mathbf{D}\left(\nabla w_{t} \nabla w^{T}\right)\right\|_{H^{1}}\left\|\nabla w_{t}\right\|_{H^{-1}} \\
& \leq c\left\|\left(\mathrm{I}-c_{\delta} \Delta\right)^{-1} \mathbf{D}\left(\nabla w_{t} \nabla w^{T}\right)\right\|_{W^{2,6 / 5}}\left\|w_{t}\right\|_{2} \leq c_{1}\left\|\nabla w_{t} \nabla w^{T}\right\|_{6 / 5}\left\|w_{t}\right\|_{2} \\
& \leq c_{1}\left\|\nabla w_{t}\right\|_{2}\|\nabla w\|_{3}\left\|w_{t}\right\|_{2} \leq c_{1}\left(\epsilon\left\|\nabla w_{t}\right\|_{2}^{2}+\frac{1}{\epsilon}\|\nabla w\|_{3}^{2}\left\|w_{t}\right\|_{2}^{2}\right)
\end{aligned}
$$

by interpolation $L^{3}=\left[L^{2} ; L^{6}\right]_{1 / 2}$

$$
\leq c_{1}\left(\epsilon\left\|\nabla w_{t}\right\|_{2}^{2}+\frac{1}{\epsilon}\|\nabla w\|_{2}\|\nabla w\|_{6}\left\|w_{t}\right\|_{2}^{2}\right)
$$

by Sobolev embedding $W^{1,2} \hookrightarrow L^{6}$ and the uniform bound for $\|\nabla w\|_{2}$

$$
\leq c_{2}\left(\epsilon\left\|\nabla w_{t}\right\|_{2}^{2}+\frac{1}{\epsilon}\left\|D^{2} w\right\|_{2}\left\|w_{t}\right\|_{2}^{2}\right)
$$

and finally, by Lemma 3.6 ,

$$
\leq c_{2}\left(\epsilon\left\|\nabla w_{t}\right\|_{2}^{2}+\frac{1}{\epsilon}\|P \Delta w\|_{2}\left\|w_{t}\right\|_{2}^{2}\right) .
$$

Now we can prove the first step for time regularity. By using equation (3.2) and inequalities (3.9)-(3.10) we get:

$$
\begin{aligned}
\frac{1}{2} \frac{d}{d t}\left\|w_{t}\right\|_{2}^{2}+\frac{1}{R e}\left\|\nabla w_{t}\right\|_{2}^{2} & \leq \frac{c}{\epsilon}\left(1+\left\|w_{t}\right\|_{2}^{4}+\|P \Delta w\|_{2}\left\|w_{t}\right\|_{2}^{2}\right)+c_{1} \epsilon\left\|\nabla w_{t}\right\|_{2}^{2} \\
& \leq \frac{c}{\epsilon}\left(1+\left\|w_{t}\right\|_{2}^{4}+\frac{\left\|w_{t}\right\|_{2}^{4}}{\epsilon^{2}}+\epsilon^{2}\|P \Delta w\|_{2}^{2}\right)+c_{1} \epsilon\left\|\nabla w_{t}\right\|_{2}^{2}
\end{aligned}
$$

and by estimate $(3.8)$

$$
\begin{aligned}
& \leq \frac{c}{\epsilon^{3}}\left(1+\left\|w_{t}\right\|_{2}^{4}\right)+c_{2} \epsilon\left(1+\left\|w_{t}\right\|_{2}^{2}+\right)+c_{1} \epsilon\left\|\nabla w_{t}\right\|_{2}^{2} \\
& \leq c(\epsilon)\left(1+\left\|w_{t}\right\|_{2}^{4}\right)+c_{1} \epsilon\left\|\nabla w_{t}\right\|_{2}^{2} .
\end{aligned}
$$

Collecting all estimates we have

$$
\frac{1}{2} \frac{d}{d t}\left\|w_{t}\right\|_{2}^{2}+\left(\frac{1}{R e}-\epsilon\right)\left\|\nabla w_{t}\right\|_{2}^{2} \leq c(\epsilon)\left(1+\left\|w_{t}\right\|_{2}^{4}\right) .
$$

Choosing $\epsilon$ small enough and setting $\left\|w_{t}(\cdot, t)\right\|_{2}^{2}=Y(t)$ we have:

$$
Y^{\prime} \leq c_{1}+c_{2} Y^{2}
$$

hence

$$
Y(t) \leq c_{3}+c_{2} \int_{\eta}^{t} Y(\tau) Y(\tau) d \tau, \quad \forall t \in\left[\eta, T^{*}\right]
$$


and, by Gronwall lemma:

$$
Y(t) \leq c \mathrm{e}^{\int_{\eta}^{t} Y(\tau) d \tau} \leq c \mathrm{e}^{\int_{\eta}^{T^{*}}\left\|w_{t}\right\|_{2}^{2} d \tau}<+\infty \quad \forall t \in\left[\eta, T^{*}\right]
$$

since, by the inductive hypothesis, $w_{t} \in L^{2}\left(\eta, T^{*} ; L^{2}(Q)\right)$. Hence, we have just proved that

$$
w_{t} \in L^{\infty}\left(\eta, T^{*} ; L^{2}(Q)\right) \cap L^{2}\left(\eta, T^{*} ; H^{1}(Q)\right) .
$$

Now we consider once again equation

$$
\frac{1}{R e} \Delta w=F+\nabla q
$$

where we set $F=w_{t}+(w \cdot \nabla) w+\nabla \cdot\left(\mathrm{I}-c_{\delta} \Delta\right)^{-1}\left(2 c_{\delta} \nabla w \nabla w^{T}\right)$. We want to prove that $F \in L^{\infty}\left(\eta, T^{*} ; L^{2}(Q)\right)$. Using the same arguments as those in estimates (3.3) and (3.4) we have:

$$
\begin{aligned}
\|(w \cdot \nabla) w\|_{2}^{2} & =\left|\int_{Q}(w \cdot \nabla) w \cdot(w \cdot \nabla) w d x\right| \leq\|w\|_{6}\|\nabla w\|_{3}\|(w \cdot \nabla) w\|_{2} \\
& \leq\|w\|_{6}\|w\|_{W^{2,2}}\|(w \cdot \nabla) w\|_{2} \leq c\|\nabla w\|_{2}\|P \Delta w\|_{2}\|(w \cdot \nabla) w\|_{2} \\
& \leq c\left(\epsilon\|(w \cdot \nabla) w\|_{2}^{2}+\frac{1}{\epsilon}\|\nabla w\|_{2}^{2}\|P \Delta w\|_{2}^{2}\right) \leq \epsilon c\|(w \cdot \nabla) w\|_{2}^{2}+\frac{c_{1}}{\epsilon}
\end{aligned}
$$

where we used also estimates (3.8), (3.11), and the first part of the inductive hypothesis. Hence, choosing $\epsilon$ small enough, we have that $(w \cdot \nabla) w \in L^{\infty}\left(\eta, T^{*} ; L^{2}(Q)\right)$. Regarding the last addend:

$$
\begin{aligned}
\left\|\nabla \cdot\left(\mathrm{I}-c_{\delta} \Delta\right)^{-1}\left(2 c_{\delta} \nabla w \nabla w^{T}\right)\right\|_{2} & \leq\left\|\left(\mathrm{I}-c_{\delta} \Delta\right)^{-1}\left(2 c_{\delta} \nabla w \nabla w^{T}\right)\right\|_{H^{1}} \\
& \leq c\left\|\left(\mathrm{I}-c_{\delta} \Delta\right)^{-1}\left(2 c_{\delta} \nabla w \nabla w^{T}\right)\right\|_{W^{2,6 / 5}} \\
& \leq c_{1}\left\|\nabla w \nabla w^{T}\right\|_{\frac{6}{5}} \leq c_{1}\|\nabla w\|_{6}^{2} \\
& \leq c_{2}\|w\|_{W^{2,2}}^{2} \leq c_{3}\|P \Delta w\|_{2}^{2} \leq c_{4} .
\end{aligned}
$$

We proved in (3.11) that $w_{t} \in L^{\infty}\left(\eta, T^{*} ; L^{2}(Q)\right)$, so $F \in L^{\infty}\left(\eta, T^{*} ; L^{2}(Q)\right)$ and, by Lemma 3.6 ,

$$
w \in L^{\infty}\left(\eta, T^{*} ; H^{2}(Q)\right) .
$$

The above result allows us to prove that $\left\|w_{t}\right\|_{W^{2,2}}$ is square integrable in time. In fact, multiplying equation (3.1) by $-\Delta w_{t}$ and integrating over $Q$, we have:

$$
\begin{aligned}
& \int_{Q} \nabla w_{t t} \cdot \nabla w_{t} d x+\frac{1}{R e}\left\|\Delta w_{t}\right\|_{2}^{2} \\
& =\int_{Q}\left(w_{t} \cdot \nabla\right) w \cdot \Delta w_{t} d x+\int_{Q}(w \cdot \nabla) w_{t} \cdot \Delta w_{t} d x \\
& \quad+\int_{Q} \nabla \cdot\left(\mathrm{I}-c_{\delta} \Delta\right)^{-1} 4 c_{\delta} \mathbf{D}\left(\nabla w_{t} \nabla w^{T}\right) \cdot \Delta w_{t} d x
\end{aligned}
$$


and we shall estimate each addend separately. By Hölder and Sobolev inequalities and (3.12) we have:

$$
\begin{aligned}
\left|\int_{Q}\left(w_{t} \cdot \nabla\right) w \cdot \Delta w_{t} d x\right| & \leq\left\|w_{t}\right\|_{3}\|\nabla w\|_{6}\left\|\Delta w_{t}\right\|_{2} \leq c\left\|w_{t}\right\|_{W^{1,2}}\|w\|_{W^{2,2}}\left\|\Delta w_{t}\right\|_{2} \\
& \leq c_{1}\left\|w_{t}\right\|_{W^{1,2}}\left\|\Delta w_{t}\right\|_{2} \leq c_{1}\left(\frac{\left\|w_{t}\right\|_{W^{1,2}}^{2}}{\epsilon}+\epsilon\left\|\Delta w_{t}\right\|_{2}^{2}\right) .
\end{aligned}
$$

By Hölder inequality, (3.12) and the inclusion $W^{2,2}(Q) \subset L^{\infty}(Q)$ we get:

$$
\begin{aligned}
\left|\int_{Q}(w \cdot \nabla) w_{t} \cdot \Delta w_{t} d x\right| & \leq\|w\|_{\infty}\left\|\nabla w_{t}\right\|_{2}\left\|\Delta w_{t}\right\|_{2} \\
& \leq c\left\|w_{t}\right\|_{W^{1,2}}\left\|\Delta w_{t}\right\|_{2} \leq c\left(\frac{1}{\epsilon}\left\|w_{t}\right\|_{1,2}^{2}+\epsilon\left\|\Delta w_{t}\right\|_{2}^{2}\right) .
\end{aligned}
$$

By Hölder and Sobolev inequalities, Agmon-Douglis-Nirenberg theorem and (3.12) we obtain:

$$
\begin{aligned}
& \left|\int_{Q} \nabla \cdot\left(\mathrm{I}-c_{\delta}\right)^{-1} 4 c_{\delta} \mathbf{D}\left(\nabla w_{t} \nabla w^{T}\right) \cdot \Delta w_{t} d x\right| \\
& \leq\left\|\nabla \cdot\left(\mathrm{I}-c_{\delta} \Delta\right)^{-1} 4 c_{\delta} \mathbf{D}\left(\nabla w_{t} \nabla w^{T}\right)\right\|_{2}\left\|\Delta w_{t}\right\|_{2} \\
& \leq\left\|\left(\mathrm{I}-c_{\delta} \Delta\right)^{-1} 4 c_{\delta} \mathbf{D}\left(\nabla w_{t} \nabla w^{T}\right)\right\|_{W^{1,2}}\left\|\Delta w_{t}\right\|_{2} \\
& \leq c\left\|\left(\mathrm{I}-c_{\delta} \Delta\right)^{-1} \mathbf{D}\left(\nabla w_{t} \nabla w^{T}\right)\right\|_{W^{2,6 / 5}}\left\|\Delta w_{t}\right\|_{2} \leq c_{1}\left\|\nabla w_{t} \nabla w^{T}\right\|_{6 / 5}\left\|\Delta w_{t}\right\|_{2} \\
& \leq c_{1}\left\|\nabla w_{t}\right\|_{2}\|\nabla w\|_{3}\left\|\Delta w_{t}\right\|_{2} \leq c_{1}\left\|\nabla w_{t}\right\|_{2}\|w\|_{2,2}\left\|\Delta w_{t}\right\|_{2} \\
& \leq c_{2}\left(\frac{1}{\epsilon}\left\|w_{t}\right\|_{W^{1,2}}^{2}+\epsilon\left\|\Delta w_{t}\right\|_{2}^{2}\right) .
\end{aligned}
$$

Substituting these estimates in equation (3.13) we obtain:

$$
\frac{1}{2} \frac{d}{d t}\left\|\nabla w_{t}\right\|_{2}^{2}+\left(\frac{1}{R e}-c \epsilon\right)\left\|\Delta w_{t}\right\|_{2}^{2} \leq \frac{c^{\prime}}{\epsilon}\left\|w_{t}\right\|_{W^{1,2}}^{2} .
$$

Choosing $\epsilon$ small enough, integrating with respect to $t$ and using (3.11), we have that $\nabla w_{t} \in L^{\infty}\left(\eta, T^{*} ; L^{2}(Q)\right)$ and $\Delta w_{t} \in L^{2}\left(\eta, T ; L^{2}(Q)\right)$. Hence, by Lemma 3.6, $w_{t} \in L^{2}\left(\eta, T^{*} ; H^{2}(Q)\right)$ and, by $(3.11)$

$$
w_{t} \in L^{\infty}\left(\eta, T^{*} ; H^{1}(Q)\right) \cap L^{2}\left(\eta, T^{*} ; H^{2}(Q)\right) .
$$

Finally, multiplying equation (3.1) by $w_{t t}$ and integrating over $Q$ we have:

$$
\begin{aligned}
\left\|w_{t t}\right\|_{2}^{2}+\frac{1}{R e} \frac{1}{2} \frac{d}{d t}\left\|\nabla w_{t}\right\|_{2}^{2}= & -\int_{Q}\left(w_{t} \cdot \nabla\right) w \cdot w_{t t} d x-\int_{Q}(w \cdot \nabla) w_{t} \cdot w_{t t} d x \\
& -\int_{Q} \nabla \cdot\left(\mathrm{I}-c_{\delta} \Delta\right)^{-1} 4 c_{\delta} \mathbf{D}\left(\nabla w_{t} \nabla w^{T}\right) \cdot w_{t t} d x
\end{aligned}
$$


We evaluate the integrals on the right hand-side separately. By (3.12), (3.15), Hölder, and Sobolev inequalities, we have:

$$
\begin{aligned}
\left|\int_{Q}\left(w_{t} \cdot \nabla\right) w \cdot w_{t t} d x\right| & \leq\left\|w_{t}\right\|_{3}\|\nabla w\|_{6}\left\|w_{t t}\right\|_{2} \leq\left\|w_{t}\right\|_{W^{1,2}}\left\|D^{2} w\right\|_{2}\left\|w_{t t}\right\|_{2} \\
& \leq c\left\|w_{t t}\right\|_{2} \leq \frac{c^{2}}{\epsilon}+\epsilon\left\|w_{t t}\right\|_{2}^{2} .
\end{aligned}
$$

By (3.12), (3.15), and Hölder inequality:

$$
\left|\int_{Q}(w \cdot \nabla) w_{t} \cdot w_{t t} d x\right| \leq\|w\|_{\infty}\left\|\nabla w_{t}\right\|_{2}\left\|w_{t t}\right\|_{2} \leq c\left\|w_{t t}\right\|_{2} \leq \frac{c^{2}}{\epsilon}+\epsilon\left\|w_{t t}\right\|_{2}^{2} .
$$

Using the same estimates as in (3.14), replacing $\Delta w_{t}$ by $w_{t t}$, we obtain that:

$$
\begin{aligned}
& \left|\int_{Q} \nabla \cdot\left(\mathrm{I}-c_{\delta} \Delta\right)^{-1} 4 c_{\delta} \mathbf{D}\left(\nabla w_{t} \nabla w^{T}\right) \cdot w_{t t} d x\right| \\
& \leq c\left(\frac{1}{\epsilon}\left\|w_{t}\right\|_{1,2}^{2}+\epsilon\left\|w_{t t}\right\|_{2}^{2}\right) \leq \frac{c_{1}}{\epsilon}+c_{2} \epsilon\left\|w_{t t}\right\|_{2}^{2} .
\end{aligned}
$$

Collecting the above results and choosing $\epsilon$ small enough, we obtain:

$$
\left\|w_{t t}\right\|_{2}^{2}+c \frac{d}{d t}\left\|\nabla w_{t}\right\|_{2}^{2} \leq c_{3}
$$

and, integrating in $t$, we obtain that $w_{t t} \in L^{2}\left(\eta, T^{*} ; L^{2}(Q)\right)$.

We now explain how the other inductive steps will follow. Complete details are left to the interested reader. Starting with the $k-1$-inductive step

$$
\begin{aligned}
D_{t}^{k-1} w & \in L^{\infty}\left(\eta, T^{*}, H^{1}(Q)\right) \cap L^{2}\left(\eta, T^{*} ; H^{2}(Q)\right) ; \\
D_{t}^{k} w & \in L^{2}\left(\eta, T^{*} ; L^{2}(Q)\right),
\end{aligned}
$$

we now (briefly) show how we can arrive at the $k$-step, for a generic $\mathbf{N} \ni k \geq 1$.

Step 1. The first step is obtained by deriving the equation $k$ times with respect to $t$. By multiplying by $D_{t}^{k} w$ we have mainly to estimate the following terms

$$
\int D_{t}^{k}[(w \cdot \nabla) w] D_{t}^{k} w d x \quad \text { and } \quad \int D_{t}^{k}\left[\nabla \cdot\left(\mathrm{I}-c_{\delta} \Delta\right)^{-1} 2 c_{\delta}\left(\nabla w \nabla w^{T}\right)\right] D_{t}^{k} w d x
$$

By following the same approach used in the previous steps and by using Leibniz differentiation rule, we can easily obtain

$$
\begin{aligned}
\left|\int D_{t}^{k}[(w \cdot \nabla) w] D_{t}^{k} w d x\right|+ & \left|\int D_{t}^{k}\left[\nabla \cdot\left(\mathrm{I}-c_{\delta} \Delta\right)^{-1} 2 c_{\delta}\left(\nabla w \nabla w^{T}\right)\right] D_{t}^{k} w d x\right| \\
& \leq \epsilon\left\|\nabla D_{t}^{k} w\right\|^{2}+c\left\|\Delta D_{t}^{k-1} w\right\|^{2}\left\|D_{t}^{k} w\right\|^{2} .
\end{aligned}
$$

In this case we are left with the differential inequality

$$
\frac{d}{d t}\left\|D_{t}^{k} w\right\|_{2}^{2}+\frac{1}{R e}\left\|\nabla D_{t}^{k} w\right\|_{2}^{2} \leq c\left\|\Delta D_{t}^{k-1} w\right\|_{2}^{2}\left\|D_{t}^{k} w\right\|^{2} .
$$


By using the results proved in the previous inductive step, the Gronwall lemma implies

$$
D_{t}^{k} w \in L^{\infty}\left(\eta, T ; L^{2}(Q)\right) \cap L^{2}\left(\eta, T ; H^{1}(Q)\right) .
$$

Step 2. The second step is obtained by deriving the equations $k$ times with respect to the time variable, by multiplying by $-\Delta D_{t}^{k} w$, and by using suitable integration by parts. The terms that need some care are the following

$$
\int D_{t}^{k}[(w \cdot \nabla) w] \Delta D_{t}^{k} w d x \quad \text { and } \quad \int D_{t}^{k}\left[\nabla \cdot\left(\mathrm{I}-c_{\delta} \Delta\right)^{-1} 2 c_{\delta}\left(\nabla w \nabla w^{T}\right)\right] \Delta D_{t}^{k} w d x .
$$

By using the same techniques we obtain the following estimate (we also used the results of the previous inductive step)

$$
\begin{aligned}
\left|\int D_{t}^{k}[(w \cdot \nabla) w] \Delta D_{t}^{k} w d x\right|+ & \left|\int D_{t}^{k}\left[\nabla \cdot\left(\mathrm{I}-c_{\delta} \Delta\right)^{-1} 2 c_{\delta}\left(\nabla w \nabla w^{T}\right)\right] \Delta D_{t}^{k} w d x\right| \\
& \leq \epsilon\left\|\Delta D_{t}^{k} w\right\|^{2}+c\left\|\Delta D_{t}^{k-1} w\right\|^{2}\left\|\nabla D_{t}^{k} w\right\|^{2} .
\end{aligned}
$$

We arrive then at the following

$$
\frac{d}{d t}\left\|\nabla D_{t}^{k} w\right\|_{2}^{2}+\frac{1}{R e}\left\|\Delta D_{t}^{k} w\right\|_{2}^{2} \leq c\left\|\Delta D_{t}^{k-1} w\right\| \nabla D_{t}^{k} w \|^{2} .
$$

By using the result proved in Step 1, the last differential inequality implies that

$$
D_{t}^{k} w \in L^{\infty}\left(\eta, T ; H^{1}(Q)\right) \cap L^{2}\left(\eta, T ; H^{2}(Q)\right) .
$$

Step 3. The last step will follow by deriving the equations $k$ times with respect to the time variable, by multiplying by $D_{t}^{k+1} w$, and by using suitable integration by parts. The terms needing some care are the following $\int D_{t}^{k}[(w \cdot \nabla) w] D_{t}^{k+1} w d x \quad$ and $\quad \int D_{t}^{k}\left[\nabla \cdot\left(\mathrm{I}-c_{\delta} \Delta\right)^{-1} 2 c_{\delta}\left(\nabla w \nabla w^{T}\right)\right] D_{t}^{k+1} w d x$

By using the same techniques we obtain the following estimate

$$
\begin{aligned}
\left|\int D_{t}^{k}[(w \cdot \nabla) w] D_{t}^{k+1} w d x\right|+ & \left|\int D_{t}^{k}\left[\nabla \cdot\left(\mathrm{I}-c_{\delta} \Delta\right)^{-1} 2 c_{\delta}\left(\nabla w \nabla w^{T}\right)\right] D_{t}^{k+1} w d x\right| \\
& \leq \epsilon\left\|D_{t}^{k+1} w\right\|^{2}+c\left\|\Delta D_{t}^{k} w\right\|^{2}\left\|\nabla D_{t}^{k} w\right\|^{2} .
\end{aligned}
$$

We arrive then at the following

$$
\left\|D_{t}^{k+1} w\right\|_{2}^{2}+\frac{d}{d t} \frac{1}{R e}\left\|\nabla D_{t}^{k} w\right\|_{2}^{2} \leq c\left\|\Delta D_{t}^{k} w\right\|^{2}\left\|\nabla D_{t}^{k} w\right\|^{2} .
$$

By using the result proved in Step 1-2, we have finally

$$
D_{t}^{k+1} w \in L^{2}\left(\eta, T ; L^{2}\right) .
$$

This concludes the inductive step. 
The inductive step has been completed, so we have proved that

$$
D_{t}^{k} w \in L^{2}\left(\eta, T^{*}, H^{2}(Q)\right) \quad \forall k \in \mathbf{N}
$$

and, by Morrey inequality $\left(W^{1, p} \subset C^{0,1-\frac{3}{p}}\right)$,

$$
D_{t}^{k} w \in C\left(\left(\eta, T^{*}\right) ; H^{2}(Q)\right) \quad \forall k \in \mathbf{N} .
$$

To show the space regularity we prove the following lemma.

Lemma 3.8. Let $w$ be a strong solution to (1.1) in $\left[0, T^{*}[\right.$. Then

$$
D_{t}^{k} \in L^{2}\left(\eta, T^{*} ; W^{m, 2}(Q)\right) \quad \forall m, k \in \mathbf{N}, \forall \eta>0 .
$$

Proof. As in the previous lemma we shall work with the Galerkin approximation for $w$. Let us consider the RLES equation derived with respect to time:

$$
\frac{1}{R e} \Delta w_{t}=w_{t t}+\left(w_{t} \cdot \nabla\right) w+(w \cdot \nabla) w_{t}+\nabla \cdot\left(\mathrm{I}-c_{\delta} \Delta\right)^{-1} 4 c_{\delta} \mathbf{D}\left(\nabla w_{t} \nabla w^{T}\right) .
$$

We want to show that the right hand-side of the above equation belongs to $L^{2}\left(\eta, T^{*} ; W^{1,2}(Q)\right)$. We already know, by $(3.16)$, that $w_{t t} \in L^{2}\left(\eta, T^{*} ; W^{1,2}(Q)\right)$; let us examine the remaining terms. Using Hölder and Sobolev inequalities we get:

$$
\begin{aligned}
\left\|\left(w_{t} \cdot \nabla\right) w\right\|_{2} & \leq\left\|w_{t}\right\|_{4}\|\nabla w\|_{4} \leq\left\|w_{t}\right\|_{W^{1,2}}\|w\|_{W^{2,2}} \\
\left\|\nabla\left[\left(w_{t} \cdot \nabla\right) w\right]\right\|_{2} & \leq\left\|\left(\nabla w_{t} \cdot \nabla\right) w\right\|_{2}+\left\|\left(w_{t} \cdot \nabla\right) \nabla w\right\|+\left\|w_{t}\right\|_{\infty}\left\|D^{2} w\right\|_{2} \\
& \leq\left\|\nabla w_{t}\right\|_{4}\|\nabla w\|_{4}+c\left\|w_{t}\right\|_{2,2}\|w\|_{2,2} \\
& \leq c_{1}\left\|w_{t}\right\|_{W^{1,4}}\|w\|_{W^{2,2}}+c\left\|w_{t}\right\|_{W^{2,2}}\|w\|_{W^{2,2}} \leq c_{2}\left\|w_{t}\right\|_{W^{2,2}}\|w\|_{W^{2,2}}
\end{aligned}
$$

hence

$$
\left\|\left(w_{t} \cdot \nabla\right) w\right\|_{W^{1,2}} \leq c\left\|w_{t}\right\|_{W^{2,2}}\|w\|_{W^{2,2}} .
$$

In a similar way we have that:

$$
\left\|(w \cdot \nabla) w_{t}\right\|_{W^{1,2}} \leq c\|w\|_{W^{2,2}}\left\|w_{t}\right\|_{W^{2,2}} .
$$

The evaluation of the last term gives us:

$$
\begin{aligned}
& \left\|\nabla \cdot\left(\mathrm{I}-c_{\delta} \Delta\right)^{-1} 4 c_{\delta} \mathbf{D}\left(\nabla w_{t} \nabla w^{T}\right)\right\|_{W^{1,2}} \\
& \leq c\left\|\left(\mathrm{I}-c_{\delta} \Delta\right)^{-1} \nabla \mathbf{D}\left(\nabla w_{t} \nabla w^{T}\right)\right\|_{W^{2,2}} \\
& \leq c\left\|\nabla w_{t} \nabla w^{T}\right\|_{2} \leq c\left\|\nabla w_{t}\right\|_{4} \nabla w\left\|_{4} \leq c\right\| \nabla w_{t}\left\|_{4}\right\| w\left\|_{W^{2,2}} \leq c\right\| w_{t}\left\|_{W^{2,2}}\right\| w \|_{W^{2,2}} .
\end{aligned}
$$

Hence, using the inequality $\left\|w_{t}\right\|_{W^{2,2}}\|w\|_{W^{2,2}} \leq \frac{1}{2}\left(\left\|w_{t}\right\|_{W^{2,2}}^{2}+\|w\|_{W^{2,2}}^{2}\right)$ and (3.16) we get that the right hand side of equation (3.17) belongs to

$$
L^{2}\left(\eta, T^{*} ; W^{1,2}(Q)\right)
$$

Now we are ready to apply Lemma 3.6 to get $w_{t} \in L^{2}\left(\eta, T^{*} ; W^{3,2}(Q)\right)$. An induction argument, whose details are left to the reader, proves the thesis. 
The thesis of Theorem 3.5 is then an easy consequence of Lemma 3.8 and Morrey inequality.

Let us now show that the previous estimates can provide global existence if the $H^{1}$ norm of the initial datum is small:

Theorem 3.9. Let $w$ be a strong solution to (1.1) with $\bar{f}=0$. If $\left\|\nabla w_{0}\right\|_{2}$ is small enough then the solution exists for every $t \in[0, T]$.

Proof. We know, from Theorems 3.1 and 3.2 that the strong solution exists in a time interval $[0, \bar{T})$ with $\bar{T}$. Let us suppose that $\bar{T}$ is the maximal existence time and that $\bar{T}<T$. By considering equation (1.1) with $\bar{f}=0$, multiplying by $-\Delta w$, integrating by parts over $Q$ and using Hölder inequality, we obtain:

$$
\begin{aligned}
& \frac{1}{2} \frac{d}{d t}\|\nabla w\|_{2}^{2}+\frac{1}{R e}\|\Delta w\|_{2}^{2} \\
& \leq\left|\int_{Q}(w \cdot \nabla) w \cdot \Delta w d x\right|+\left|\int_{Q} \nabla \cdot\left(\mathrm{I}-c_{\delta} \Delta\right)^{-1} 2 c_{\delta}\left(\nabla w \nabla w^{T}\right) \cdot \Delta w d x\right| \\
& \leq\|w\|_{6}\|\nabla w\|_{3}\|\Delta w\|_{2}+\left\|\nabla\left(\mathrm{I}-c_{\delta} \Delta\right)^{-1} 2 c_{\delta}\left(\nabla w \nabla w^{T}\right)\right\|_{2}\|\Delta w\|_{2}
\end{aligned}
$$

using Sobolev embeddings $W^{1,2} \hookrightarrow L^{6}$ and $W^{2, \frac{6}{5}} \hookrightarrow W^{1,2}$ :

$$
\leq c\left(\|\nabla w\|_{2}\|\nabla w\|_{3}\|\Delta w\|_{2}+\left\|\left(\mathrm{I}-c_{\delta}\right)^{-1} \nabla w \nabla w^{T}\right\|_{W^{2, \frac{6}{5}}}\|\Delta w\|_{2}\right)
$$

by using the Agmon-Douglis-Nirenberg regularity results (see [1, 2]):

$$
\leq c\left(\|\nabla w\|_{2}\left\|\nabla w_{6}\right\|\|\Delta w\|_{2}+\left\|\nabla w \nabla w^{T}\right\|_{\frac{6}{5}}\|\Delta w\|_{2}\right)
$$

by using Sobolev embedding $W^{2,2} \hookrightarrow W^{1,6}$, Lemma 3.6 and Hölder inequality:

$$
\leq c\left(\|\nabla w\|_{2}\|\Delta w\|_{2}^{2}+\|\nabla w\|_{\frac{12}{5}}^{2}\|\Delta w\|_{2}\right)
$$

using the interpolation result $L^{\frac{12}{5}}=\left[L^{2} ; L^{3}\right]_{\frac{1}{2}}$, the Sobolev embedding $W^{2,2} \hookrightarrow$ $W^{1,3}$ and Lemma 3.6:

$$
\leq c\left(\|\nabla w\|_{2}\|\Delta w\|_{2}^{2}+\|\nabla w\|_{2}\|\nabla w\|_{3}\|\Delta w\|_{2}\right) \leq c\|\nabla w\|_{2}\|\Delta w\|_{2}^{2} .
$$

Hence, if $\left\|\nabla w_{0}\right\|_{2} \leq \frac{1}{c R e}$, we get

$$
\frac{1}{2} \frac{d}{d t}\|\nabla w\|_{2}^{2}+\frac{1}{R e}\|\Delta w\|_{2}^{2} \leq 0
$$

and the norm $\|\nabla w\|_{2}$ results not increasing. Then, there would exist a sequence $\left\{t_{k}\right\}_{k \in \mathbf{N}}$ (that satisfies $t_{k} \uparrow \bar{T}$ ) such that

$$
\left\|\nabla w\left(t_{k}\right)\right\| \leq\left\|\nabla w_{0}\right\| .
$$


Since $w\left(t_{k}\right) \in H^{1}$, by using Theorem 3.1 we may construct a solution $\bar{w}$ with initial datum $w\left(t_{k}\right)$ in a time interval $\left[t_{k}, t_{k}+T^{*}\right)$, where $T^{*}$ depends on $\left\|\nabla w_{0}\right\|$, and $R e$. By using the uniqueness of such strong solution, we have $w \equiv \bar{w}$ in $\left[t_{k}, t_{k}+T^{*}\right)$. We may now select $k_{0} \in \mathbf{N}$ such that $t_{k_{0}}+T^{*}>\bar{T}$ to contradict the assumption on the maximality of $\bar{T}$. This proves Theorem 3.9.

\section{Some exact solutions for benchmarking}

In this section we recall some classical results on explicit solutions to the NavierStokes equations and we show how these can lead to explicit solution to the Rational Large Eddy Simulation model (1.1), too. In particular, we want to point out that solutions that are periodic, and that share particular symmetries annihilate exactly the additional nonlinear term (1.3). This is the basic observation of this section. Most of the results that we will use can be found in the classical paper by Berker [6] and also in the celebrated book by Lamb [32], while recent (computationally oriented) results are those by Ross Ethier and Steinman [34]. To make formulæ of this section more readable, in this section we suppose that $c_{\delta}:=\delta^{2} / 24=1$. The reader can derive similar results for different values of the regularizing parameter $\delta$. The positive constant Re denotes again the Reynolds number.

\subsection{Planar flow}

First we consider the classical stationary, planar flow

$$
u_{1}=A y^{2}+B y+C, \quad u_{2} \equiv 0, \quad p=\frac{2 A}{R e} x+p_{0},
$$

for arbitrary constants $A, B, C \in \mathbf{R}$. This leads to some well known solutions, as the Poiseuille flow (see Lamb [32]); or the flow over an inclined plane, with a free surface. The latter can be also used to design a device that allows to measure the kinematic viscosity of a given fluid, see Berker $[6], \S 6$. In the case of the above planar flow (4.1) the additional part of the stress tensor - the one coming from (1.3) - becomes

$$
2 \operatorname{div}(\mathrm{I}-\Delta)^{-1}\left(\begin{array}{cc}
(2 A y+B)^{2} & 0 \\
0 & 0
\end{array}\right)
$$

In this case we did not specify appropriate boundary conditions for the inversion of the Laplace operator, yet, but if we consider the portion of a two dimensional channel

$$
\mathcal{C}:=\{(x, y): 0 \leq x \leq L,-1 \leq y \leq 1\},
$$

it could be natural to take the homogeneous Dirichlet boundary conditions on the solid walls $y= \pm 1$, while to impose a Neumann condition at the ends $x=0$, 
$x=L$. The first component of the solution is then

$$
u_{1}(y)=A\left(1-y^{2}\right)
$$

while the second vanishes identically. In this case the inversion of the operator leads to the following tensor

$$
\operatorname{div}\left(\begin{array}{cc}
8 A+4 A y^{2}+C_{1} \sinh (y)+C_{2} \cosh (y) & 0 \\
0 & 0
\end{array}\right) \equiv\left(\begin{array}{l}
0 \\
0
\end{array}\right) .
$$

In this particular case it is possible to make explicit calculations on the solution and to verify that it is independent of $x$. Since the solution is unique, we found the only solution to such problem. In this way we found that the Poiseuille flow could be an explicit solution also for the RLES model.

Note that the inversion with different boundary conditions can imply that the resulting function is no longer independent of $x$, and the above reasoning cannot work further.

\subsection{Taylor solutions}

Regarding the two dimensional case, it is worth noting that there exists a wide class of explicit non-stationary solutions to the Navier-Stokes equations, called Taylor solutions [37]. These solutions are defined via the stream function $\psi$ as follows

$$
u_{1}=-\frac{\partial \psi}{\partial x_{2}} \quad \text { and } \quad u_{2}=\frac{\partial \psi}{\partial x_{1}}
$$

where $\psi$ satisfies

$$
\psi(x, y, t)=\mathrm{e}^{k t / R e} F(x, y),
$$

with $k$ an arbitrary constant (it will turn out to be an eigenvalue of the Laplacian), while $F$ is a solution to the equation

$$
\Delta F=k F .
$$

Concerning this solution we have several interesting observations, see Berker [6], $\S 39$ for further details:

- This is a non-stationary solution in which the current lines do not change their shape; furthermore, they coincide with the trajectories. At each point the direction of the velocity remains the same, while its modulus is not constant.

- Unsteady terms balance viscous terms in the momentum equation.

- The convective term can be expressed as a gradient of a scalar (the negative of the pressure).

The solution originally considered by Taylor is that corresponding to the following stream function:

$$
\psi(x, y, t)=A \mathrm{e}^{-\frac{2 \pi^{2} t}{a^{2} R e}} \cos \left(\frac{\pi x}{a}\right) \sin \left(\frac{\pi y}{a}\right) .
$$


This stream function, if $a=A=1$, leads to the following exact solution:

$$
\left\{\begin{aligned}
u_{1} & =-\cos (\pi x) \sin (\pi y) \mathrm{e}^{-2 \pi^{2} t / R e} \\
u_{2} & =\sin (\pi x) \cos (\pi y) \mathrm{e}^{-2 \pi^{2} t / r e} \\
p & =-\frac{\cos (2 \pi x) \sin (2 \pi y)}{4} \mathrm{e}^{-4 \pi^{2} t / R e}
\end{aligned}\right.
$$

and this solution has been used as a benchmark in the well-known simulations performed by Kim and Moin [30].

We consider the above solution rewritten in a slightly different form:

$$
\left\{\begin{aligned}
u_{1} & =-\sin (\pi y) \mathrm{e}^{-\pi^{2} t / R e} \\
u_{2} & =\sin (\pi x) \mathrm{e}^{-\pi^{2} t / R e} \\
p & =-\cos (\pi x) \sin (\pi y) \mathrm{e}^{-2 \pi^{2} t / R e}
\end{aligned}\right.
$$

The solution (4.3) can be obtained by the Taylor solution, after a rotation of angle $\pi / 4$, an homothety of scale factor $\sqrt{2} / 2$, and a translation of the origin. The two solution are essentially the same and they are solve the Navier-Stokes equations (1.1) in the square $[0,2] \times[0,2]$.

If we consider the above exact solution (4.3) to the Navier-Stokes equations, the additional stress tensor becomes:

$$
2 \operatorname{div}(\mathrm{I}-\Delta)^{-1}\left(\begin{array}{cc}
\pi^{2} \cos ^{2}(\pi y) & 0 \\
0 & 0
\end{array}\right) \mathrm{e}^{-4 \pi^{2} t / R e}
$$

In this case the operator $(\mathrm{I}-\Delta)^{-1}$ has to be considered as supplemented with periodic boundary conditions. Resorting to the Fourier expansion we look for periodic functions $\phi$, such that $\phi-\Delta \phi=f$ with $f=\pi^{2} \cos ^{2}(\pi y)$, or $f=0$. Such solutions can be expressed in the following form

$$
\phi(x, y)=\sum_{l, m \in \mathbf{Z}^{2}} c_{l m} \mathrm{e}^{i \pi(l x+m y)},
$$

and it is easy to see that the coefficients with $l \neq 0$ must vanish, since the entries of the matrix do not depend on $x$. The terms corresponding to an identically zero right hand side must vanish for the same reasoning and stemming to the uniqueness of such solutions, we finally arrive at the following expression for the additional (turbulent stress tensor) term in (1.1):

$$
\operatorname{div}\left(\begin{array}{cc}
\phi(y) \mathrm{e}^{-2 \pi^{2} t / R e} & 0 \\
0 & 0
\end{array}\right) \equiv\left(\begin{array}{l}
0 \\
0
\end{array}\right) .
$$

We have finally proved that (4.3) is a suitable solution to RLES model, too. It is easily computable and useful for benchmarking or debugging complex codes. 


\subsection{Three dimensional solutions}

In this section, by using the same ideas of the previous one, we provide an explicit 3D solution for the RLES equation. The methods comes from the generalization of the Taylor solution given by Ross Ethier and Steinman [34]. The idea is to employ the same technique used by Taylor to produce a vector-valued generalization $\Psi$ of the stream function, i.e., a vector field $\Psi=\left(\Psi_{y z}, \Psi_{z x}, \Psi_{x y}\right)$ such that:

$$
\Delta \boldsymbol{\Psi}(x, y, z)=\lambda^{2} \boldsymbol{\Psi}(x, y, z) .
$$

Then, the exact solution results the following

$$
u(x, y, z, t)=\mathbf{V}(x, y, z) T(t)=\nabla \times \mathbf{\Psi}(x, y, z) \mathrm{e}^{\lambda^{2} t},
$$

where $\lambda$ is a complex constant. The solution turns out to be expressed as follows

$$
\left\{\begin{array}{l}
\Psi_{y z}=f(y) g(z) h(x) \\
\Psi_{z x}=f(z) g(x) h(y) \\
\Psi_{x y}=f(x) g(y) h(z),
\end{array}\right.
$$

with

$$
f^{\prime \prime}=a^{2} f, \quad g^{\prime \prime}=b^{2} g, \quad h^{\prime \prime}=c^{2} h,
$$

and $a^{2}+b^{2}+c^{2}=\lambda^{2}$. The flow obtained is a particular case of the generalized Beltrami flows (introduced by Beltrami [5]), i.e., flows such that

$$
\nabla \times(\mathbf{V} \times(\nabla \times \mathbf{V}))=\mathbf{0}
$$

and consequently the term $(\mathbf{V} \cdot \nabla) \mathbf{V}$ can be expressed as a gradient.

We also note that the $2 \mathrm{D}$ Taylor solution is recovered by setting $\Psi_{y z}=\Psi_{z x}=0$ and $h=1$.

We consider a particular form of this generalized Beltrami flow, namely

$$
\left\{\begin{aligned}
& u_{1}=[A \sin (\pi z)+C \cos (\pi y)] \mathrm{e}^{-\pi^{2} t / R e} \\
& u_{2}=[B \sin (\pi x)+A \cos (\pi z)] \mathrm{e}^{-\pi^{2} t / R e} \\
& u_{3}=[C \sin (\pi y)+B \cos (\pi x)] \mathrm{e}^{-\pi^{2} t / R e} \\
& p=-[B C \cos (\pi x) \sin (\pi y)+A B \sin (\pi x) \cos (\pi z) \\
&\quad+A C \sin (\pi z) \cos (\pi y)] \mathrm{e}^{-2 \pi^{2} t / R e},
\end{aligned}\right.
$$

where $A, B$, and $C$ are arbitrary constants. Straightforward calculations show that (4.4) is an exact solution of the Navier-Stokes equations (1.2) and that it belongs to the class of Trkal flows (see Berker [6], § 50), i.e., flows such that

$$
\exists c \in \mathbf{R}: \quad \nabla \times u=c u .
$$

(In this particular case $c=1$.) 
Velocity is not constant in time, but the current lines coincide with trajectories, for (4.4), too. The qualitative behavior is similar to that of Taylor solutions. The main difference is that in Taylor solutions, the vorticity is perpendicular to the velocity, while in Trkal solutions the vorticity is aligned with the velocity field.

The flow (4.4) turns out to be the viscous unsteady counterpart of the ABCArnol'd-Beltrami-Childress flow (see [4] and [11]) that has been introduced to study the instabilities and the possible singularities of the solutions to the Euler equations.

Explicit calculations for the "turbulent part" of the stress tensor show that

$$
\operatorname{div}(\mathrm{I}-\Delta)^{-1}\left(\nabla u \nabla u^{T}\right) \equiv\left(\begin{array}{l}
0 \\
0 \\
0
\end{array}\right) .
$$

The calculation are similar to those given in the $2 \mathrm{D}$ case. If $i=1,2,3$ and $x_{1}=x$, $x_{2}=y, x_{3}=z$, then, for $i, j=1,2,3$ :

$$
\left(\nabla u \nabla u^{T}\right)_{i, j}=F_{i j}\left(x_{k}\right) \mathrm{e}^{-2 \pi^{2} t / R e}, \quad \text { with } k \neq i .
$$

$\left(F_{i j}\left(x_{k}\right)\right.$ denotes a generic function only of $x_{k}$.) Again, the fact that the solution of the problem with the inverse of I $-\Delta$ must be found in the space of periodic functions, allows us to show (4.5). This implies that (4.4) is an explicit solution to the 3D RLES equations, too.

Remark 4.1. The classes of exact solutions we have found are also exact solutions to the Gradient model (2.2). The same explicit calculations, without the inversion of the Laplacian, show how (4.3) and (4.4) are also exact solution to (2.2).

\section{Results of some numerical experiments}

In this section we show the results of some numerical experiments we performed. We studied the problem in the space periodic setting and consequently we used the pure Fourier-Galerkin method.

\subsection{The numerical method}

The approximate solution is obtained by discretization of the space variable with periodic functions, i.e., we look for solutions in the following form

$$
u=\sum_{k \in \mathbf{Z}^{2}} c_{k} \mathrm{e}^{\frac{2 i \pi k \cdot x}{\mathcal{L}}}, \quad \bar{c}_{k}=c_{-k}, \quad c_{0}=0, \quad k \cdot c_{k}=0
$$

and we consider a truncation of this vector field. Let the operator $P^{m}$ denotes the orthogonal projection in the subspace generated by the functions $\left\{\mathrm{e}^{\frac{2 i \pi k \cdot x}{\mathcal{L}}}\right\}_{|k| \leq m}$, 
with $|k|=\max k_{i}$. By considering a proper variational formulation, we solve the following system of ordinary differential equations

$$
\left\{\begin{aligned}
& \frac{\partial w^{m}}{\partial t}+\nabla q^{m}+P^{m}(\nabla \cdot(w \otimes w))-\frac{1}{R e} \Delta w^{m} \\
&+P^{m}\left\{\nabla \cdot\left(\mathrm{I}-\frac{\delta^{2}}{24} \Delta\right)^{-1}\left[\frac{\delta^{2}}{12} \nabla w \nabla w^{T}\right]\right\}=P^{m} \bar{f} \\
& \nabla \cdot w^{m}=0 \\
& w^{m}(x, 0)=P^{m} w^{0}(x),
\end{aligned}\right.
$$

where $w^{m}$ denotes the truncation of $w$ at the level $m$. To discretize the time variable we use an explicit Adams-Bashfort two-step method, see for instance Iserles [26], $\S 2$. We use it in conjunction with a small enough temporal step, to obtain stability of the method.

The codes were run over a multi-processor PentiumIII CPU with the standard glibc libraries and the maximum number of Fourier modes we were allowed to consider was $64^{2}$; with these inputs we could get a reasonable computational time, at moderate Reynolds numbers.

We were mainly interested in the calculation of the kinetic energy, to compare the decreasing behavior expected by the solution of the Navier-Stokes equations (1.2) (in the case of a vanishing external force) with unknown one of the kinetic energy of the solutions to the RLES model (1.1). In fact, this model may allow backscatter of energy, improving performances of eddy viscosity models, see Fischer and Iliescu [24].

In this periodic setting the inversion of the operator

$$
\left(\mathrm{I}-\frac{\delta^{2}}{24} \Delta\right)
$$

is particularly simple, since it turns out to be an algebraic operation, when performed in the Fourier space. This is why we think that the RLES model may well perform with spectral methods. The implementation of more sophisticated spectral methods will be the object of a future paper. The results of this paper are not conclusive, but of interest for the study of flows that are simple, though not laminar. Again, we furnish test cases that can be employed to debug complex fully $3 \mathrm{D}$ codes.

\subsection{Some test cases with a vanishing external force}

We considered a zero external force, and we took as initial datum a function that is similar to the exact solutions we introduced in the previous section. The initial 
datum we considered is (after projection by means of $P^{m}$ ) the following

$$
u:=\left\{\begin{array}{l}
u_{1}=\cos (2 \pi(y+\cos (2 \pi y))+\cos (2 \pi y) \\
u_{2}=\sin (2 \pi x) .
\end{array}\right.
$$

In fact, note that if we remove in $u_{1}$ the first entry (cf. (4.3) at $t=0$ ) we have one of the exact solutions introduced in the previous section. The term we added is smooth and periodic, but its Fourier development is not trivial since it has an infinite number of non-vanishing terms.

We considered the problem with different Reynolds numbers, but in any case we used moderated ones. To have stability of the numerical solutions we could not really study turbulent flows and this is outside the scope of the present paper.

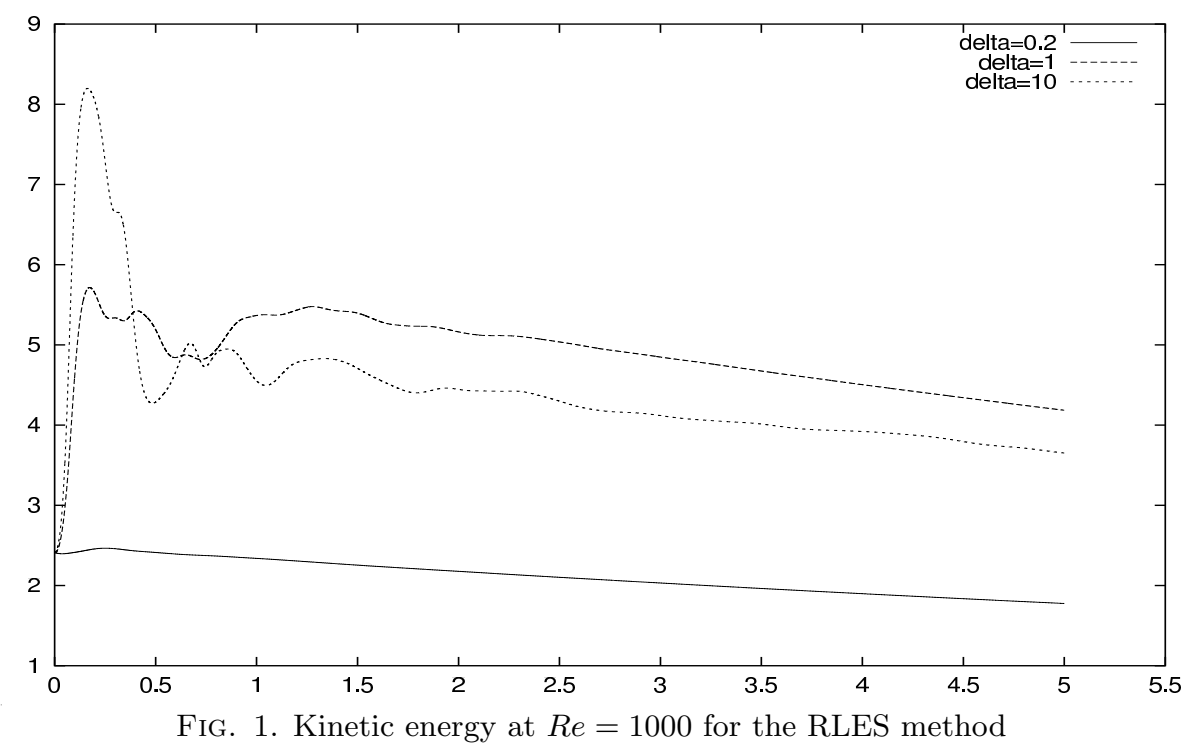

The analysis of flows with very high Reynolds numbers would require too big computational efforts and we are interested to see how the RLES model performs in some special and simple situations. See also Berselli [7] for different perspectives in the numerical treatment of some LES methods.

We mainly focused on the behavior of the kinetic energy of the solution, with respect to the averaging parameter $\delta$. Figure 1 shows the effect of a moderate $\delta$. We note that the "optimal" value for $\delta$ is about $1 / 10$, since in this case the mesh size and the scale of the filter almost coincide. In this case the behavior of the energy is very similar to that of the Navier-Stokes equations.

If we choose a too big value for $\delta$ we see that - after some transient - the energy decreases, provided the Reynolds number is not too high. We can see that if the Reynolds number is not too high, then the effect of the added term seems not to 
influence too much the behavior of the solution. Further analysis at high Reynolds is needed to understand the effect of the RLES term on the kinetic energy and some transient instabilities are reported in preliminary tests.

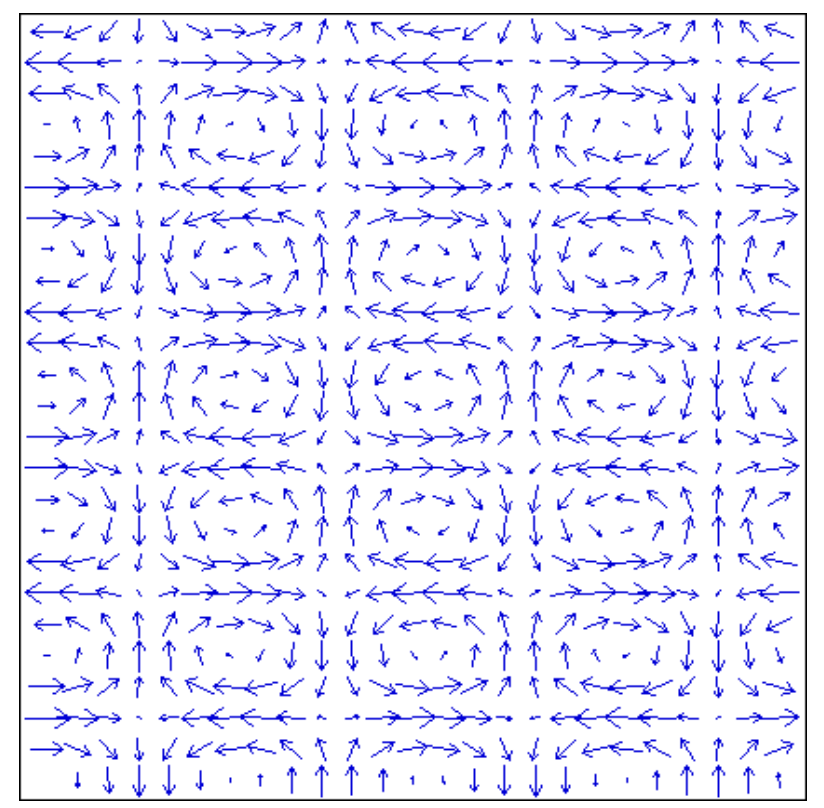

FIG. 2. 2D Taylor velocity field, computed with RLES model, $t=1 \mathrm{sec}$

Another test we performed is that starting with

$$
\left\{\begin{array}{l}
u_{1}=-\cos (\pi x) \sin (\pi y) \\
u_{2}=\sin (\pi x) \cos (\pi y)
\end{array}\right.
$$

as initial datum (it is (4.2) at $t=0$ ). In this case the RLES model produces a field that is very close to the exact 2D Taylor solution: the velocity goes to zero while the streamlines remain essentially the same during all the computational time, as shown in Fig. 2.

\subsubsection{Comparison with the Gradient model}

To compare the RLES model with the Gradient model, we implemented the same algorithm, by running almost the same code (obviously without the inversion of I $-\Delta$ ) on the Gradient model (2.2) to see if the solutions obtained with this method are less stable, as reported in several references, see for instance Coletti [13] and Iliescu et al. [25]. In particular, we observed finite-time blow up for the kinetic energy, if a strong stabilization is not added. 
We also performed some simulations by starting with the 2D-Taylor solution (4.2). In the case of the RLES we have seen that this solution is recovered very well. On the other hand the Gradient model produces very big instabilities and the vorticity, instead of being confined in an array of vortices, seems to spread out all over the computational domain. Even when the kinetic energy remains bounded, the solution that is calculated is very far from the exact one. We believe that this is due to nonlinear instability of the Gradient model. We can see the result of our computations, where we plotted the streamlines, and also the value of the vorticity.

In Figures 3 and 4 we plotted respectively the following data:

upper left: velocity field lower left: streamlines upper right: vorticity level sets lower right: vorticity.
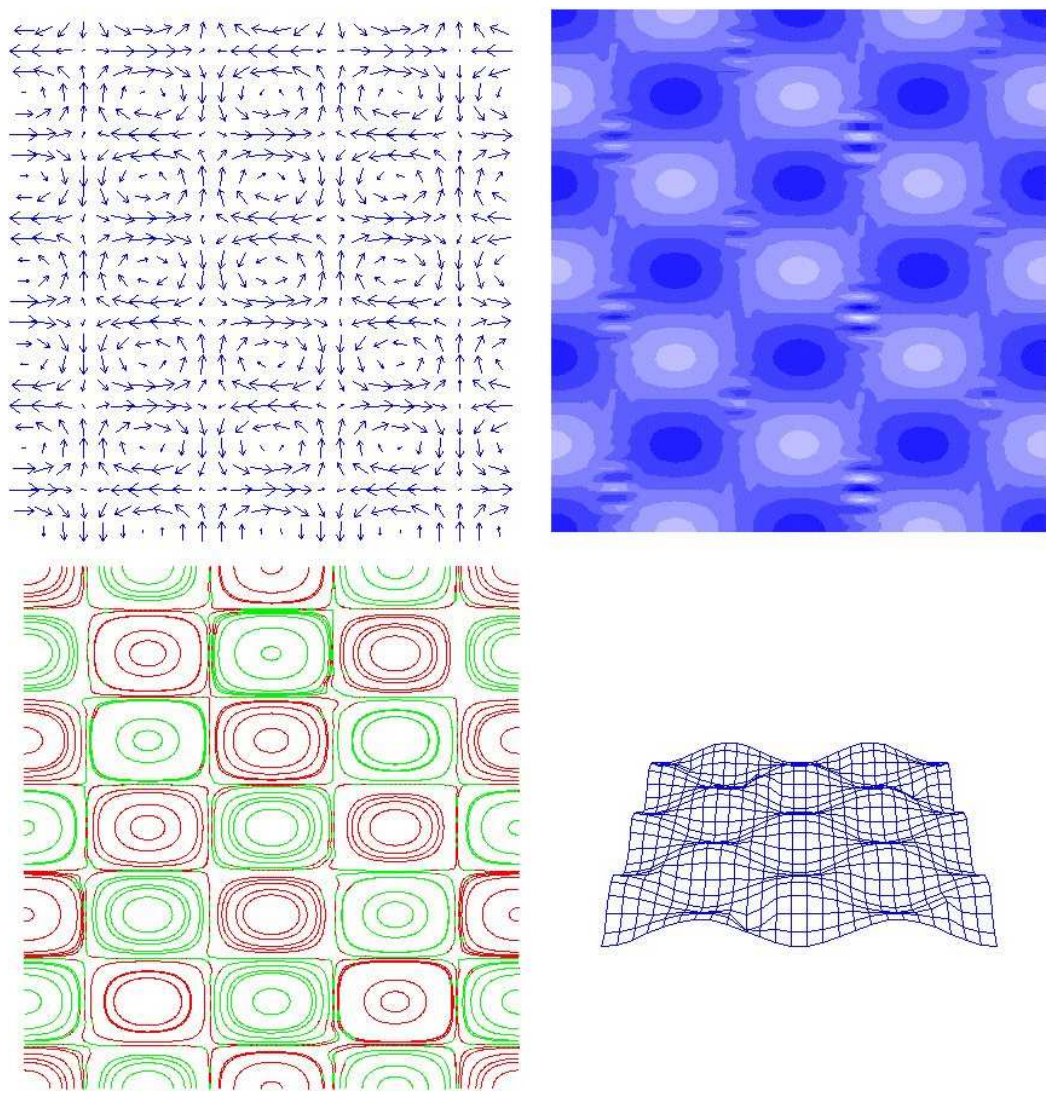

FIG. 3. Gradient model, $R e=1000, t=0.056 \mathrm{~s}$ 

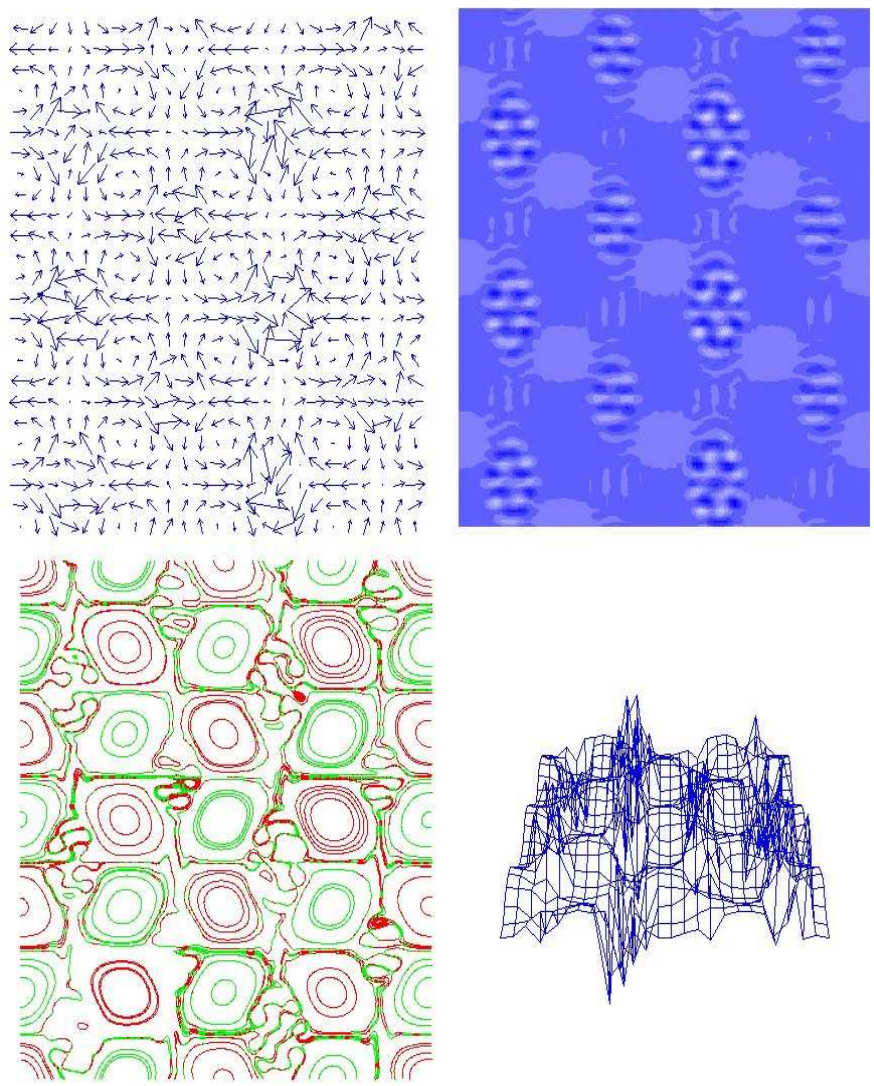

FiG. 4. Gradient model, $R e=1000, t=0.062 \mathrm{~s}$

\subsection{A test case with external forcing}

We also tested the RLES model with an external force. In this case the solution is driven by the force to a stationary solution. We run our code on the following test case: consider the equations with a vanishing initial datum, but with an external force $f$ concentrated in two balls

$$
B_{i}=\left\{(x, y) \in[0,1]^{2}: \sqrt{\left(x-P_{i x}\right)^{2}+\left(y-P_{i y}\right)^{2}}<\frac{1}{8}\right\}
$$

where

$$
\underline{P_{i}}:=\left(P_{i x}, P_{i y}\right)=\left(\frac{1}{2}+\frac{1}{4} \cos (0.7), \frac{1}{2}+(-1)^{i+1} \frac{1}{4} \sin (0.7)\right) .
$$

If we denote by $\left(r_{i}, \phi_{i}\right)$ the polar coordinates with origin in $\underline{P_{i}}$, inside the ball $B_{i}$ we have the force

$$
f_{i}\left(r_{i}, \phi_{i}\right)=\left(f_{r_{i}}, f_{\phi_{i}}\right)^{T}=\left(0, f_{\phi_{i}}\right)^{T},
$$


where

$$
f_{\phi_{i}}:=\frac{(-1)^{i+1}}{8 r_{i}} \int_{0}^{r_{i}} \rho(1+\cos (4 \rho))^{2} d \rho .
$$

The force $f:[0,1]^{2} \rightarrow \mathbf{R}^{2}$ we shall consider is then the following: if $f_{i}$ denotes the function defined by (5.1)-(5.2), then

$$
f:= \begin{cases}f_{1} & \text { in } B_{1} \\ f_{2} & \text { in } B_{2}, \\ 0 & \text { otherwise }\end{cases}
$$

In each ball the force has no components in the radial direction, but is always directed in the tangential direction. Consequently the force drives the flow to rotate around the points $P_{i}$, to generate two big vortices. This behavior is confirmed by our simulation, and we also compared the solution, with that one obtained from the Navier-Stokes equations, i.e., by setting $\delta=0$.

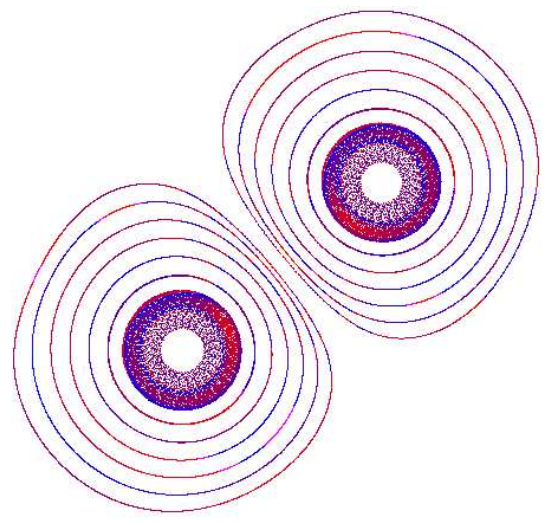

FIG. 5. Streamlines at $R e=1000, \delta=0$

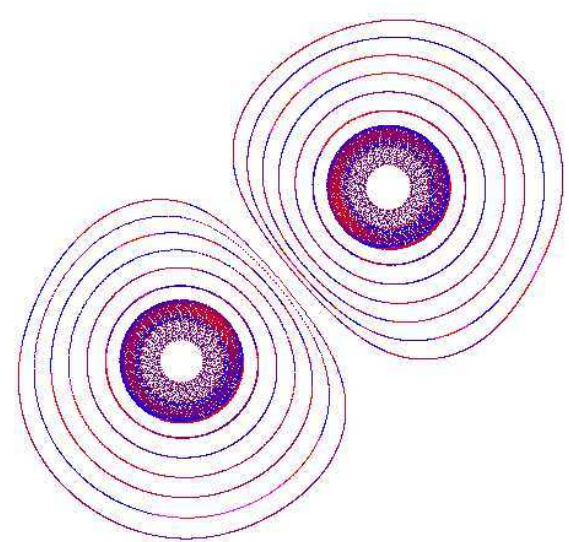

FIG. $6 . R e=1000, \delta=0.1$

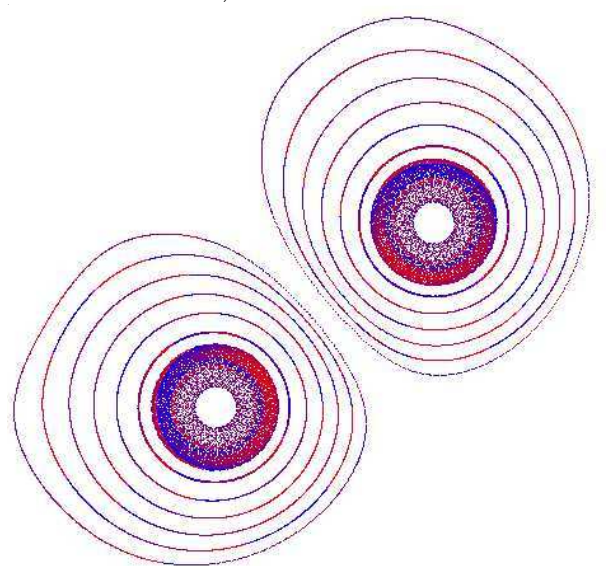

Fig. 7. $R e=1000, \delta=1$ 


\section{References}

[1] S. Agmon, A. Douglis and L. Nirenberg, Estimates near the boundary for solutions of elliptic partial differential equations satisfying general boundary conditions. I, Comm. Pure Appl. Math. 12 (1959), 623-727.

[2] S. Agmon, A. Douglis and L. Nirenberg, Estimates near the boundary for solutions of elliptic partial differential equations satisfying general boundary conditions. II, Comm. Pure Appl. Math. 17 (1964), 35-92.

[3] A. A. Aldama, Filtering techniques for turbulent flow simulation, Springer-Verlag, Berlin, 1990.

[4] V. Arnol'd, Sur la topologie des écoulements stationnaires des fluides parfaits, C. R. Acad. Sci. Paris 261 (1965), 17-20.

[5] E. Beltrami, Sui principii fondamentali dell'idrodinamica razionale, Mem. dell'Accad. Scienze Bologna (1873), 394.

[6] R. Berker, Intégration des équations du mouvement d'un fluide visqueux incompressible, in: Handbuch der Physik, Bd. VIII/2, Springer, Berlin, 1963, pp. 1-384.

[7] L. C. Berselli, On the large eddy simulation of the Taylor-Green vortex, J. Math. Fluid Mech. 7 (2005), S164-S191.

[8] L. C. Berselli, G. P. Galdi, T. Iliescu and W. J. Layton, Mathematical analysis for the rational large eddy simulation model, Math. Models Methods Appl. Sci. 12 (2002), 1-22.

[9] L. C. Berselli and C. R. Grisanti, On the consistency of the rational large eddy simulation model, Comput. Vis. Sci. 6 (2004), 75-82.

[10] L. C. Berselli and T. Iliescu, A higher order subfilter-scale model for large eddy simulation, J. Comput. Appl. Math. 159 (2003), 411-430.

[11] S. Childress, New Solutions of the kinematics dynamo problem, J. Math. Phys. 11 (1970), 3063-3076.

[12] P. Coletti, A global existence theorem for large eddy simulation turbulence model, Math. Models Methods Appl. Sci. 7 (1997), 579-591.

[13] P. Coletti, Analytical and numerical results for $k-\epsilon$ and large eddy simulation turbulence models, Phd thesis, UTM PhDTS 17, Università di Trento, Italy, 1998.

[14] G.-H. Cottet, D. Jiroveanu and B. Michaux, Vorticity dynamics and turbulence models for Large-Eddy Simulations, M2AN Math. Model. Numer. Anal. 37 (2003), 187-207.

[15] A. Dunca, V. John and W. J. Layton, The commutation error of space averaged NavierStokes equations on a bounded domain, in: Contributions to current challenges in mathematical fluid mechanics, 53-78, Adv. Math. Fluid Mech. Birkhäuser, Basel, 2004.

[16] C. FoiAs, D. D. Holm and E. S. Titi, The Navier-Stokes-alpha model of fluid turbulence, Phys. D 152/153 (2001), 505-519, Advances in nonlinear mathematics and science.

[17] G. P. Galdi and W. J. Layton, Approximation of the larger eddies in fluid motions. II. A model for space-filtered flow, Math. Models Methods Appl. Sci. 10 (2000), 343-350.

[18] B. J. Geurts, Elements of Direct and Large-Eddy Simulation, R. T. Edwards, Inc., 2003.

[19] T. J. R. Hughes, L. Mazzei, D. D. Holm, J. E. Marsden and T. S. Ratiu, Euler-Poincaré models of ideal fluids with nonlinear dispersion, Phys. Rev. Lett. 80 (1998), 4173-4176.

[20] T. J. R. Hughes, L. Mazzei and K. E. Jansen, Large eddy simulation and the variational multiscale method, Comput. Visual. Sci. 3 (2000), 47-59.

[21] T. Iliescu, Large eddy simulation for turbulent flows, Ph.D. thesis, Department of Mathematics, Pittsburgh University, Pittsburgh, PA, 2000.

[22] T. IliesCu and P. Fischer, A 3d channel flow simulation at $R e_{\tau}=180$ using a rational LES model, in: L. Sakell, C. Liu and T. Beutner (eds.), Proceedings of Third AFOSR International Conference on DNS/LES, 283-290, 2001.

[23] T. Iliescu and P. Fischer, Large eddy simulation of turbulent channel flows by the rational LES model, Phys. Fluids 15 (2003), 3036-3047.

[24] T. Iliescu and P. Fischer, Backscatter in the Rational LES model, Comput. \& Fluids 35 (2004), 783-790.

[25] T. Iliescu, V. John, W. J. Layton, G. Matthies and L. Tobiska, A numerical study of a class of LES models, Int. J. Comput. Fluid. Dyn. 17 (2003), 75-85. 
[26] A. Iserles, A first course in the numerical analysis of differential equations, Cambridge Texts in Applied Mathematics, Cambridge University Press, Cambridge, 1996.

[27] V. John, Large eddy simulation of turbulent incompressible flows. Analytical and numerical results for a class of LES models, Lecture Notes in Computational Science and Engineering, Springer-Verlag, Berlin, 2004.

[28] V. John, W. J. LAYTon and N. SAhin, Derivation and analysis of near wall models for channel and recirculating flows, Comput. Math. Appl. 28 (2004), 1135-1151.

[29] F. V. Katopodes, R. L. Street and J. H. Ferziger, A theory for the subfilter-scale model in large-eddy simulation, EFML Technical Report 2000-K1, Stanford University, 2000.

[30] J. KIM and P. MoIN, Application of a fractional-step method to incompressible NavierStokes equations, J. Comput. Phys. 59 (1985), 308-323.

[31] O. A. LADYŽHENSKAYA, New equations for the description of the motions of viscous incompressible fluids, and global solvability for their boundary value problems, Trudy Mat. Inst. Steklov 102 (1967), 85-104.

[32] H. LAmB, Hydrodynamics, reprint of the 1932 sixth edition, sixth ed., Cambridge University Press, Cambridge, 1993. (With a foreword by R. A. Caflisch.)

[33] W. J. Layton, Analysis of a scale-similarity model of the motion of large eddies in turbulent flows, J. Math. Anal. Appl. 264 (2001), 546-559.

[34] C. Ross Ethier and D. A. Steinman, Exact fully 3D Navier-Stokes solutions for benchmarking, Internat. J. Numer. Methods Fluids 19 (1994), 369-375.

[35] P. Sagaut, Large eddy simulation for incompressible flows, Scientific Computation, Springer-Verlag, Berlin, 2001. With an introduction by Marcel Lesieur. Translated from the 1998 French original by the author.

[36] S. Stolz and N. A. AdAms, An approximate deconvolution procedure for large-eddy simulation, Phys. Fluids 11 (1999), 1699-1701.

[37] G. I. Taylor, On decay of vortices in a viscous fluid, Phil. Mag. (6) 46 (1923), 671-674.

David Barbato

Scuola Normale Superiore

Piazza dei Cavalieri

Pisa

Italy

e-mail: barbato@sns.it
Luigi C. Berselli and Carlo R. Grisanti Dipartimento di Matematica Applicata "U. Dini" Università di Pisa

V. Bonanno 25/b

Pisa

Italy

e-mail: berselli@dma.unipi.it grisanti@dma.unipi.it

(accepted: February 28, 2005)

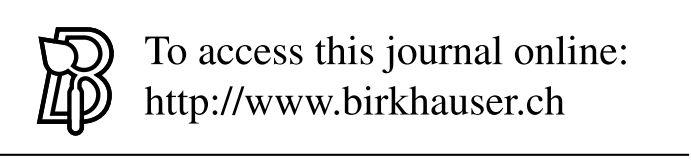

